\title{
Altered Neural Processing of the Need to Stop in Young Adults at Risk for Stimulant Dependence
}

\author{
Katia M. Harlé, ${ }^{1}$ Pradeep Shenoy, ${ }^{2}$ Jennifer L. Stewart, ${ }^{1}$ Susan F. Tapert, ${ }^{1,3}$ Angela J. Yu, ${ }^{2 \star}$ and Martin P. Paulus ${ }^{1,3 *}$ \\ ${ }^{1}$ Department of Psychiatry and ${ }^{2}$ Department of Cognitive Science, University of California San Diego, La Jolla, California 92093, and ${ }^{3}$ Psychiatry Service, VA \\ San Diego Healthcare System, La Jolla, California 92161
}

Identification of neurocognitive predictors of substance dependence is an important step in developing approaches to prevent addiction. Given evidence of inhibitory control deficits in substance abusers (Monterosso et al., 2005; Fu et al., 2008; Lawrence et al., 2009; Tabibnia et al., 2011), we examined neural processing characteristics in human occasional stimulant users (OSU), a population at risk for dependence. A total of 158 nondependent OSU and 47 stimulant-naive control subjects (CS) were recruited and completed a stop signal task while undergoing functional magnetic resonance imaging (fMRI). A Bayesian ideal observer model was used to predict probabilistic expectations of inhibitory demand, $P($ stop), on a trial-to-trial basis, based on experienced trial history. Compared with CS, OSU showed attenuated neural activation related to $P($ stop) magnitude in several areas, including left prefrontal cortex and left caudate. OSU also showed reduced neural activation in the dorsal anterior cingulate cortex (AACC) and right insula in response to an unsigned Bayesian prediction error representing the discrepancy between stimulus outcome and the predicted probability of a stop trial. These results indicate that, despite minimal overt behavioral manifestations, OSU use fewer brain processing resources to predict and update the need for response inhibition, processes that are critical for adjusting and optimizing behavioral performance, which may provide a biomarker for the development of substance dependence.

Key words: Bayesian model; cognitive control; ideal observer model; inhibitory control; stimulants

\section{Introduction}

Inhibitory control, the ability to withhold a prepotent action, is an important cognitive control process and is hypothesized to be dysfunctional in individuals with substance use problems. However, it is unclear how dysfunctional cognitive control progresses with continuing substance use. Here, we combine Bayesian ideal observer model-based analysis with fast, event-related fMRI data to investigate subtle behavioral and neural differences between occasional stimulant users (OSU), a population at risk for developing dependence (Tapert et al., 2002; Elkashef and Vocci, 2003), and healthy control subjects (CS).

Prior investigations have shown inhibitory control deficits in stimulant-dependent individuals, with moderate correlations with drug use indices (Simon et al., 2002; Fillmore and Rush, 2002; Salo et al., 2002; Monterosso et al., 2005; Hester et al., 2007; Tabibnia et al., 2011). Stimulant dependence has been linked to reduced functioning of dopamine transporters and hypometabolism in various regions critical to inhibitory control, including basal ganglia, anterior cingulate cortex (ACC), and other pre-

\footnotetext{
Received May 29, 2013; revised Jan. 28, 2014; accepted Feb. 18, 2014.

Author contributions: M.P.P. designed research; K.M.H. and P.S. analyzed data; K.M.H., P.S., J.L.S., S.F.T., A.J.Y., and M.P.P. wrote the paper.

This work was supported by the National Institutes of Health (Grant R01 DA016663-01A1).

Correspondence should be addressed to Martin Paulus, Laboratory of Biological Dynamics and Theoretical Medicine, Department of Psychiatry, University of California San Diego, 8939 Villa La Jolla Drive, Suite 200, La Jolla, CA 92037-0985.E-mail:mpaulus@ucsd.edu.

*A.J.Y. and M.P.P. are cosenior authors.

DOI:10.1523/JNEUROSCI.2297-13.2014

Copyright $\odot 2014$ the authors $\quad 0270-6474 / 14 / 344567-14 \$ 15.00 / 0$
}

frontal areas (Volkow et al., 1999; Bolla et al., 2004; London et al., 2004; Kim et al., 2009). During inhibitory control tasks, primarily go/no-go and Stroop paradigms, cocaine abusers also show hypoactivity in the ACC, pre-SMA, superior frontal gyrus, and insula (Kaufman et al., 2003; Hester and Garavan, 2004; Li et al., 2008). In contrast to dependent users, there are relatively few studies on occasional users, although some behavioral studies suggest subtle impairments in inhibitory response and error monitoring (Colzato et al., 2007; Reske et al., 2011).

Previously, we showed that healthy volunteers continuously alter their response strategy in a stop-signal task, a classical inhibitory control paradigm, such that dynamic fluctuations in their reaction time and error rate are consistent (Ide et al., 2013) with a particular Bayesian sequential adjustment algorithm (Yu and Cohen, 2009) and decision strategy (Shenoy and Yu, 2011; for analogous results in primates, see Emeric et al., 2007; Scangos and Stuphorn, 2010; Stuphorn et al., 2010). A Bayesian approach (Yu and Cohen, 2009; Ide et al., 2013) is used here to identify any difference between OSU and healthy CS in their neural representation of probabilistic expectations and the incorporation of prediction errors needed for updating those expectations. Specifically, we test the hypothesis that inhibitory dysfunction in OSU is characterized by a decreased ability to represent or respond to the likelihood of encountering an inhibitory stimulus. Therefore, we expect them to have both an altered representation of the expectation of an inhibitory signal and an altered prediction error signal; that is, the discrepancy between prediction and outcome, which is critical for adjusting expectations. Given recent work 
implicating the dorsal ACC in coding prediction error associated with stimulus and action outcome (Ide et al., 2013), we hypothesize OSU to have specific impairments in this region when tracking prediction errors.

\section{Materials and Methods}

Participants

The study protocol was approved by the University of California-San Diego Human Subjects Review Board and all subjects gave written informed consent. A total of 158 (62 female) nondependent OSU and 47 (26 female) stimulant naive CS were recruited over a period of 5 years via flyers mailed to $>7000$ students at local universities, internet ads (e.g., Craigslist), and local university newspapers. OSU were defined as having the following characteristics: (1) at least 2 off-prescription uses of cocaine or prescription stimulants (amphetamines and/or methylphenidate) over the past 6 months, (2) no evidence for lifetime stimulant dependence, (3) no lifetime use of stimulants for medical reasons, and (4) absence of treatment of substance- or alcohol-related problems. The following exclusion criteria were applied for all subjects: (1) current (and past 6 months) of the following Axis I diagnoses: panic disorder, social phobia, posttraumatic stress disorder, major depressive disorder; (2) lifetime bipolar disorder, schizophrenia, or obsessive compulsive disorder; (3) antisocial personality disorder (ASPD) or conduct disorder; (4) attention deficit hyperactivity disorder (ADHD); (5) current positive urine toxicology test (exception due to long detectability in urine: marijuana); (6) lifetime use of ecstasy $>25$; and (7) head injuries or loss of consciousness for $>5$ min. Additional inclusion criteria for CS were no lifetime use of stimulants and no lifetime history of substance- or alcohol-related dependence.

Lifetime DSM-IV Axis I diagnoses (including ADHD and substance abuse and dependence according to the American Psychological Association, 1994) and Axis II ASPD diagnoses were assessed by the Semistructured Assessment for the Genetics of Alcoholism (Bucholz et al., 1994). Diagnoses were based on consensus meetings with a clinician specialized in substance use disorders (M.P.P). During the interview session, subjects also performed the Wechsler Test of Adult Reading (Wechsler, 2001), a measure of verbal IQ. In addition, information on current alcohol and nicotine use patterns was collected. Several personality measures were also collected, including the Sensation Seeking Scale (SSS; Zuckerman and Link, 1968), the Barratt Impulsiveness Scale (BIS; Barratt, 1959), and the Temperament and Character Inventory (TCI; Cloninger et al., 1994). The Reward Dependence Scale of the TCI, which was included in our analyses, is a self-report inventory measuring responsiveness to signals of reward and social approval, ranging from low motivation/high social detachment to high motivation to pursue reward/high sensitivity to social cues. Higher scores on this scale are associated with persistent rewardseeking behaviors that are susceptible to emotional influences and have been associated with prefrontal hypoactivations (Sugiura et al., 2000) and lower basal noradrenergic activity (Cloninger, 1986).

\section{Stop signal task}

At the onset of each trial, either an "X" or an "O" appeared on a black background back projected to the MRI room. Participants were instructed to press, as quickly as possible, the left button when an " $\mathrm{X}$ " appeared, and the right button when an "O" appeared. They were also instructed not to press either mouse button whenever they heard a tone during a trial (Stop trials). Each trial lasted $1300 \mathrm{~ms}$ and each trial was separated by $200 \mathrm{~ms}$ interstimulus intervals (blank screen; see Matthews et al., 2005). Individual response latency was used to denote the period of inhibitory processing and provided a subject-dependent jittered reference function. Participants performed six blocks of the task, each containing a total of 48 trials ( 12 stop and 36 nonstop trials in each block). Trial order was pseudorandomized throughout the task and counterbalanced. Before scanning, participants performed the stop task in a behavioral testing session to determine their mean reaction time (RT) from " $\mathrm{X}$ " and "O" stimuli onset. Such individual measures were used to determine the stop signal delay (SSD) for the six different stop trial types. Specifically, stop signals were delivered at 0 (RT-0), 100 (RT-100), 200 (RT-200), 300 (RT-300), 400 (RT-400), or 500 (RT-500) ms less than the mean RT after the beginning of the trial, thus providing a range of difficulty level.

\section{Stop signal reaction times computation}

To estimate subject-specific time course of response inhibition, we computed the stop signal reaction times (SSRTs) for each participant, a measure that can be conceptualized as the average latency of inhibition (Logan and Cowan, 1984). SSRT can be computed by subtracting the SSD at which participants correctly inhibited button press $50 \%$ of the time from their mean RT on Go trials (mean go RT $-\mathrm{SSD}_{50 \%}$; Aron et al., 2003; Chambers et al., 2006; Li et al., 2006). To obtain this measure, we first fitted individual 2-parameter logistic equations to binary error data $(0,1)$ as a function of SSD (Hanes and Schall, 1995; Verbruggen and Logan, 2009) based on the following equation:

$$
\mathrm{P}(\text { error })=1 /\left(1+\mathrm{e}^{-(\mathrm{a}+\mathrm{bX})}\right)
$$

We then calculated $\mathrm{SSD}_{50 \%}$ by solving the equation for $x$ with $\mathrm{P}(\mathrm{e})=0.5$, which is equivalent to the median of the inhibitory function and represents the time delay between go and stop signals that a subject would need to succeed in $50 \%$ of the Stop trials. Individual SSRTs were computed by subtracting $\mathrm{SSD}_{50 \%}$ from mean go RT.

\section{Bayesian model of probabilistic prediction}

We previously proposed a Bayes-optimal model for stopping behavior (Shenoy and Yu, 2011) that decides whether and when to generate a response in the stop signal task via an evolving, moment-by-moment decision process influenced by the accumulation of noisy sensory evidence, as well as by costs associated with various actions and outcomes. In particular, the mean stop error rate and the go RT trade off against each other such that a more conservative (more hesitant) policy for responding would result in both longer RT on Go trials and lower error rate on Stop trials and vice versa for a less conservative (less hesitant) policy for responding (Shenoy and Yu, 2011). This tradeoff is influenced by factors such as the prior probability of a stop signal (Shenoy and Yu, 2011). The within-trial model predicts a linear increase in RT associated with a linear increase in the probability of a stop trial (Ide et al., 2013). In previous work (Shenoy and Yu, 2011; Shenoy et al., 2011; Ide et al., 2013), we showed that trial-by-trial adjustments of behavior in the task are well captured by a Bayesian inference and decision-making model, which assumes that the subject continually updates the prior probability of encountering Stop trials, $P$ (stop), on a trial-by-trial basis, based on trial history (Yu and Cohen, 2009) and that the subject adjusts his/her decision policy as a function of $P$ (stop), with systematic consequences for go $\mathrm{RT}$ and stop accuracy in the upcoming trial. In particular, a higher predicated $P$ (stop) leads to both a slower go RT and a higher likelihood of correctly stopping on a stop trial (Ide et al., 2013).

Briefly, to model the trial-by-trial adjustment of prior expectations, we adapted the Dynamic Belief Model (Yu and Cohen, 2009; Ide et al., 2013) as follows: the stop signal frequency $r_{k}$ on trial $k$ has probability $\alpha$ of being the same as $r_{k-1}$ and probability $1-\alpha$ of being resampled from a prior $\beta$ distribution $p_{0}(r)$. The probability of trial $k$ being a stop trial, $P_{\mathrm{k}}($ stop $)=P\left(s_{k}=1 \mid \mathrm{S}_{\mathrm{k}-1}\right)$, where $\mathrm{S}_{\mathrm{k}}=\left(s_{1}, \ldots, s_{k}\right)$ is 1 on Stop trials and 0 on Go trials, can be computed as follows:

$$
\begin{aligned}
P\left(s_{k}=1 \mid \mathrm{S}_{\mathrm{k}-1}\right)=\int P\left(s_{k}=1 \mid r_{k}\right) p\left(r_{k} \mid \mathrm{S}_{\mathrm{k}-1}\right) d r_{k}=\int r_{k} p\left(r_{k} \mid \mathrm{S}_{\mathrm{k}-1}\right) d r_{k} & \\
& =\left\langle r_{k} \mid \mathrm{S}_{\mathrm{k}-1}\right\rangle .
\end{aligned}
$$

The predictive probability of seeing a stop trial, $P_{\mathrm{k}}$ (stop), is the mean of the predictive distribution $p\left(r_{k} \mid S_{k-1}\right)$, which is a mixture of the previous posterior distribution and a fixed prior distribution, with $\alpha$ and $1-$ $\alpha$ acting as the mixing coefficients, respectively:

$$
p\left(r_{k} \mid S_{\mathrm{k}-1}\right)=\alpha p\left(r_{k-1} \mid S_{\mathrm{k}-1}\right)+(1-\alpha) p_{0}\left(r_{k}\right)
$$

with the posterior distribution being updated according to Bayes' rule:

$$
p\left(r_{k} \mid S_{\mathrm{k}}\right) \propto P\left(S_{k} \mid r_{k}\right) p\left(r_{k} \mid S_{\mathrm{k}-1}\right)
$$

In the present study, parameters for the $\beta$ distribution $p_{0}(r)$ and $\alpha$ were kept constant across all subjects and set based on previous optimal sim- 
ulations representative of subjects' expectations in the task [i.e., $\beta(2.5,7.5$; mean $=0.25]$ and $\alpha=0.8$ (Shenoy and Yu, 2011; Ide et al., 2013). Based on these parameters and given the sequence of observed Stop/Go trials (pseudorandomized, thus here constant across subjects), we computed the corresponding sequence of subjective $P$ (stop) probabilities for the trial sequence participants experienced. In subsequent fMRI analyses, the trial-by-trial estimation of $P($ stop $)=\left\langle r_{k}\right\rangle$ (i.e., representing the most up-to-date estimated likelihood of encountering a stop signal based on all previous trials) was used as a parametric regressor in subsequent $\mathrm{fMRI}$ analyses.

We note that we first investigated whether the model predictions would be sensitive to parameters $\alpha$ and the prior distribution over $r$ at the individual level. Specifically, we tested the following parameter ranges: $\alpha \in[0.7,0.95]$ and, for the $\beta$ distribution, $m \in[0.1,0.35]$, and $s \in[5,15]$ for the mean and scale parameters, respectively, where $m=a /(a+b)$ and $s=(a+b)$ and $a$ and $b$ are the reported shape parameters. Based on this wide range of parameters, we found that the produced $P$ (stop) values were highly correlated across parameter settings and relative to those calculated with the shared group settings for $\left(r>0.9 ; R^{2}>0.8\right)$. For this reason, and due to the paucity of data for accurate parameter estimation, we chose a fixed setting following the experiment design and selected a single parameter setting for the Bayesian model based on our previous work studying the stop signal task in healthy controls (Ide et al., 2013). Subsequent analyses revealed that CS and OSU did not differ in the positive linear relationship between RT and $P$ (stop), as well as for sequential effects (i.e., post-stop slowing; see Results); we therefore used the same parameter setting for both users and controls.

\section{fMRI image acquisition and analysis}

Using a fast event-related fMRI design, each scanning session was conducted on a $3 \mathrm{~T}$ General Electric scanner $\left(\mathrm{T} 2{ }^{*}\right.$-weighted EPI, TR $=2000$ $\mathrm{ms}, \mathrm{TE}=40 \mathrm{~ms}, 64 \times 64$ matrix, $304 \mathrm{~mm}$ axial slices, $\mathrm{FOV}=220 \times 220$ $\mathrm{mm}$, in-plane voxel size $=3.437$, flip angle $=90^{\circ}$ ). Each run was acquired in sessions of 256 repetitions and lasted $8 \mathrm{~min}$ and $32 \mathrm{~s}$. Functional MRI volume acquisitions were time locked to task onset. During the same experimental session, a T1-weighted image (MPRAGE, TR $=11.4 \mathrm{~ms}$, $\mathrm{TE}=4.4 \mathrm{~ms}$, flip angle $=10$ degree, $\mathrm{FOV}=256 \times 256,1 \mathrm{~mm}^{3}$ voxels $)$ was obtained for anatomical reference.

Preprocessing. All structural and functional image processing and analysis was performed with the Analysis of Functional Neuroimages (AFNI) software package (Cox, 1996). MRI x-y slices were reconstructed into AFNI BRIK format. To minimize motion artifact, the central point of the temporal region with the largest span of fewest voxelwise outliers was used as the base for registration, adjusting all other time points in $\mathrm{dx}, \mathrm{dy}$, $\mathrm{dz}$, as well as roll, pitch, and yaw directions to align remaining images to the base image. Automated coregistration of the functional echoplanar image to the anatomical image was performed and a new outlier file was generated to determine whether additional time points should be censored based on whether a given time point greatly exceeded the mean number of voxel outliers for the time series.

First-level analyses. Three types of trials were distinguished (Go, Stop Success/SS, and Stop Error/SE) and entered in a general linear model (GLM), which were convolved with a canonical hemodynamic response function. Each of these predictors was entered both as linear regressors [multiplied by the mean of the computed $P$ (stop) probabilities across all trials] and parametrically modulated (Büchel et al., 1998) by trial-level $P($ stop) estimates. Therefore, after deconvolution, the first-level model included six task regressors [three categorical: Go, SS, SE; three parametric: Go $\times P_{\mathrm{k}}$ (stop), SS $\times P_{\mathrm{k}}$ (stop), $\mathrm{SE} \times P_{\mathrm{k}}$ (stop)], baseline and linear drift regressors, and three motion regressors (pitch, yaw, roll; Matthews et al., 2005). Finally, to control for activation-related attention and arousal processes, we included go RTs (for Go trials) and SSD (for Stop trials) as additional parametric regressors of no interest. The baseline regressor consisted of intertrial intervals and instruction phases. Images were spatially filtered using a Gaussian spatial filter (full width at half maximum $4 \mathrm{~mm}$ ) to account for individual anatomical differences. Anatomical images were manually Talairached and echoplanar images were transformed into Talairach space.
Second-level analyses. $P$ (stop) modulated activation from the first-level GLM was subjected to a voxelwise linear mixed effects (LME) analysis (Pinheiro et al., 2011), with Group (OSU, CS) and trial type [Go $\times$ $P_{\mathrm{k}}$ (stop), SS $\times P_{\mathrm{k}}$ (stop), SE $\times P_{\mathrm{k}}$ (stop)] as fixed effects and subjects treated as random effect. For the main analyses, we isolated $P$ (stop) modulated activations for Go vs Stop trials (SS and SE were averaged). Statistical maps were obtained for the group main effect and the group $X$ trial type interaction. We conducted an additional LME contrast on Stop trials only, comparing $P$ (stop) modulated activation for SS versus SE trials, and obtained statistical maps for the group $\times$ trial type interaction. Finally, the same contrast analyses were conducted for categorical regressors (Go vs Stop and SS vs SE). To correct for multiple comparisons, we used a cluster threshold adjustment based on Monte Carlo simulations (generated with AFNI's AlphaSim program), based on whole-brain voxel size and $4 \mathrm{~mm}$ smoothness. A minimum cluster volume of $384 \mu \mathrm{l}$ with a cluster significance of $p=0.01$ corrected for multiple comparisons (voxelwise probability: $p<0.005$ ).

We first report the group main effect, reflecting areas tracking prior $P$ (stop) value (regardless of trial outcome) that are differentially activated between group. The next section addresses brain areas surviving whole brain analysis for a significant interaction between clinical group (OSU vs CS) and P(stop) modulated stimulus outcome (Stop vs Go), thus identifying areas exhibiting differential $P$ (stop) activation for Go versus Stop trials (i.e., an average Bayesian prediction error representing the magnitude of the discrepancy between predicted probability of stop trial occurrence and actual trial type; Ide et al., 2013). Average activation signal was then extracted from these areas and plotted for each type of parametrically modulated trial regressor [Go $\times P($ stop $)$ and Stop $\times$ $P($ stop) ] and each group (OSU vs CS; OSU being further distinguished by drug preference, i.e., cocaine, prescription, or no preference). In this analysis, we distinguished signed and unsigned prediction errors because they may provide different types of information to adjust behavior. For example, unsigned prediction errors can be conceptualized as the overall degree of discrepancy between one's internal model prediction and actual outcome and should be thus informative about the goodness of fit of one's internal predictive model (in this case, $P$ (stop) estimation). In contrast, signed prediction errors provide additional information on the direction of these prediction/outcome discrepancies, which may be more relevant to orienting or motivating the individual toward specific actions (e.g., go vs stop). To more specifically disambiguate the type of prediction error (e.g., signed vs unsigned) associated with areas encoding a significant group by $P$ (stop) modulated trial type, we first examined the sign of these activations in CS using the following rationale. A positive or negative average percent signal change associated with $P($ stop) indicate, respectively, a positive or negative correlation between activation in this area and $P$ (stop) for a given condition (stop vs go). In contrast, percent signal change not significantly different from zero (based on one sample $t$ test) was interpreted as an absence of correlation between $P$ (stop) and neural activation. Therefore, we identified areas with nonzero activations of the same sign on Go and Stop trials (i.e., both negatively or positively correlated to $P$ (stop) on both types of trial) as reflecting a signed Bayesian prediction error. This is because, for a signed prediction error (SPE) defined as outcome $-P($ stop $)$, the $\mathrm{SPE}=0-P($ stop $)=-P$ (stop) on Go trials (because the probability of a trial being a Stop trial is zero on Go trials), whereas the SPE $=1-P$ (stop) on Stop trials (because the probability of a trial being a Stop trial is 1 on Stop trials). Therefore, activation correlated with SPE should be negatively correlated with $P$ (stop) on both types of trials. Similarly, a pattern of activation showing positive correlations with $P$ (stop) on both Go and Stop trials would indicate areas encoding -(outcome $-P$ (stop)); that is, showing neural activations proportional to $P$ (stop) - outcome, or deactivations proportional to outcome $-P($ stop $)$. This is because $-($ outcome $-P($ stop $))=P($ stop $)-$ outcome $=P($ stop $)-0=P($ stop $)$ on Go trials and $=P($ stop $)-1$ on Stop trials, thus positively correlated with $P$ (stop) for both types of trials.

In contrast, areas showing activation positively correlated to $P$ (stop) on Go trials and negatively correlated to $P$ (stop) on Stop trials were identified as areas encoding an unsigned prediction error (UPE). This is because an UPE defined as $\mid$ outcome $-P($ stop $) \mid$ is equal to $\mid 0-P($ stop $) \mid=$ $P$ (stop) on Go trials and is equal to $\mid 1-P($ stop $) \mid=1-P($ stop $)$ on Stop 
trials (because $P($ stop) values are $>0$ and $<1$ and thus $1-P$ (stop) is always $>0$ ). Therefore, activation correlating with an UPE should be positively correlated with $P$ (stop) on Go trials but negatively correlated with $P$ (stop) on Stop trials. In addition, we note that areas showing the opposite pattern (negative correlation to $P$ (stop) on Go trials and positive correlation on Stop trials) are consistent with regions showing neural activations proportional to a negative UPE (i.e., $-\mid$ outcome $-P($ stop) $\mid$ ) or deactivations proportional to UPE.

Supplemental prediction error analyses. To more accurately compare group differences in brain activations modulated by a signed or unsigned Bayesian prediction error, we conducted two additional analyses. First, we conducted a nonparametric analysis by creating a second GLM in which Go and Stop trials were grouped into low and high $P$ (stop) values [median split of estimated $P$ (stop) over all trials]. Therefore, in this model, we had four event-related regressors [Go/Low $P$ (stop), Go/High $P$ (stop), Stop/Low $P$ (stop), and Stop/High $P$ (stop)], in addition to baseline, linear drift, motion regressors parameters, and RTs as covariates of no interest. The second GLM was used to extract and plot signal change associated with these different events from the interaction clusters identified in the LME analyses (see Second-level analyses, above).

In addition, we created a third GLM with trialwise Bayesian SPE (outcome $-P($ stop $))$ and UPE | outcome $-P($ stop $) \mid$ included as parametric regressors of interest. In this model, we also included a regressor modeling trial error $(0=$ correct or $1=$ error $)$ to control for performance error related activity, as well as baseline, linear drift, motion, and RT as regressors of no interest. For second-level analysis, we subjected SPE- and UPE-modulated activation each to a voxelwise LME with Group (including user preference) modeled as fixed effect and Subject as a random effect. We restricted these analyses to the interaction clusters previously identified in the main analyses. These analyses allowed us to confirm the regions identified as showing group differences in SPE and UPE and to plot the magnitude of such modulation for each group.

Anatomical regions of interest analyses. Finally, given prior evidence suggesting that the right inferior frontal gyrus (IFG) and subthalamic nucleus (STN) play a key role in the implementation of response inhibition in the Stop Signal task (Aron et al., 2004), and because such areas were not found to be differentially activated between groups in response to $P$ (stop) and prediction error magnitude, we conducted a priori region of interest (ROI) analyses in these two regions to assess their role in Bayesian Stop signal prediction error computations and to identify any group differences in recruiting these regions. We used anatomical masks to extract activation from these ROIs, namely the right IFG (whole anatomical region from Talairach atlas) and right STN (spherical ROI of $4 \mathrm{vx}$ radius, center TC: $-13,-10,0$; based on Aron et al., 2004). For significant clusters, average percent signal change from baseline was extracted for each condition of interest.

Relationship with clinical data. To examine the potential relationship between the obtained group-dependent BOLD activations and clinical indices, we conducted voxelwise Huber regression analyses, a form of robust regression providing a more reliable estimate of correlational associations. This method is less sensitive to the effect of outliers than traditional least-square estimates and is generally more robust to the violation of traditional linear regression assumptions (e.g., multicollinearity, non-normally distributed data). Importantly, this regression strategy allowed us to assess the independent effect of each potential predictor of interest on the brain activation patterns observed within OSU (including both stimulus independent activation to $P$ (stop) and stimulus outcome-dependent activation, i.e., prediction error signal). For example, given the high usage of marijuana in OSU, we wanted to ensure that any relationship between brain activation and stimulant use measures could not be explained by marijuana use. Therefore, four regression models were tested in whole brain analyses and submitted to the same cluster-based thresholding method used for LME analyses to correct for multiple comparisons (minimum cluster volume of $384 \mu \mathrm{l}$ with a cluster significance of $p=0.01$ ). The first two were concerned with the group main effect and predicted $P$ (stop) BOLD contrast averaged across Go and Stop trials. One model included three personality questionnaire measures as predictors (i.e., BIS, SSS, and TCI Reward Dependence scale), whereas the second model included three drug use measures (i.e.,
Table 1. Subject characteristics as a function of group status $(n=205)$

\begin{tabular}{|c|c|c|c|c|c|}
\hline & \multicolumn{2}{|c|}{$\operatorname{OSU}(n=158)$} & \multicolumn{2}{|c|}{$\operatorname{CS}(n=47)$} & \multirow[b]{2}{*}{$t$ test } \\
\hline & Mean & SD & Mean & SD & \\
\hline \multicolumn{6}{|l|}{ Demographics } \\
\hline Age & 20.8 & 1.5 & 21.0 & 2.2 & $p=0.46$ \\
\hline Education & 14.6 & 1.3 & 14.6 & 1.5 & $p=0.94$ \\
\hline Verbal IQ (WTAR) & 108.9 & 7.3 & 110.0 & 7.1 & $p=0.37$ \\
\hline Alcohol (typical drinks/week) & 20.9 & 15.2 & 4.8 & 3.5 & $p<0.001$ \\
\hline Alcohol (typical days/week) & 3.2 & 1.6 & 1.5 & 0.7 & $p<0.001$ \\
\hline Nicotine (typical cigarettes/d) & 2.6 & 4.2 & 0.9 & 4.1 & $p<0.001$ \\
\hline Nicotine (typical days/week) & 2.6 & 3.2 & 0.4 & 1.5 & $p<0.05$ \\
\hline \multicolumn{6}{|l|}{ Lifetime drug use } \\
\hline Cocaine & 22.8 & 37.5 & 0.0 & 0.0 & N/A \\
\hline Prescription stimulant & 28.2 & 70.4 & 0.0 & 0.0 & $\mathrm{~N} / \mathrm{A}$ \\
\hline Marijuana & 906.4 & 1372.0 & 23.2 & 89.7 & $p<0.001^{a}$ \\
\hline
\end{tabular}

WTAR, Wechsler Test of Adult Reading; N/A, not applicable.

${ }^{a} t$ test computed using natural log-transformed +0.5 values (due to non-normal distributions) replicated results for raw data.

lifetime cocaine, prescription stimulant, and marijuana use times, each $\log$ transformed given positively skewed distributions). The other two models were concerned with the interaction of group and Bayesian prediction error and thus predicted the Stop $\times P($ stop $)-$ Go $\times P$ (stop) contrast, with the same sets of predictors used for the main effect regression models. Finally, for each set of models (main effect and interaction), we used the obtained robust Huber regression statistical maps to determine any clusters overlapping with regions identified in LME analyses.

\section{Results}

\section{Subject characteristics}

OSU did not differ from CS in ethnicity $\left(\chi^{2}(4)=8.2, p=0.08\right)$, age, education, or verbal IQ, but had more males (61\%) than CS $\left(43 \%, \chi^{2}(1)=4.2, p=0.04\right)$. In addition, OSU endorsed greater cocaine, prescription stimulant, and marijuana intake and used alcohol and nicotine more frequently and in larger quantities than CS. Within the OSU group, 47 reported predominantly using cocaine (cocaine comprised at least $80 \%$ of their lifetime stimulant use), 57 reported a preferred use of prescription stimulants ( $>80 \%$ of their lifetime stimulant use), and the remaining OSU $(n=54)$ reported similar use of both cocaine and prescription stimulants. A total of 41 OSU met criteria for marijuana dependence (thereof 18 for current dependence), whereas 46 OSU and three CS met criteria for lifetime marijuana abuse (with 110 OSU but no CS reporting using marijuana within the week of the fMRI session). In addition, 69 OSU and three CS reported regular use of nicotine at inclusion into the study (see Table 1 for detailed sample and drug use characteristics). Given these results, we examined the potential effect of sex, marijuana use (low, i.e., $<50$ lifetime uses, vs high, i.e., $>500$ lifetime uses), and stimulant preference (cocaine, i.e., cocaine comprising $80 \%$ lifetime stimulant use; prescription, i.e., prescription stimulants comprising $80 \%$ lifetime stimulant use; and no preference, i.e., remaining subgroup of OSU using both cocaine and prescription stimulants, $>30 \%$ and $<70 \%$ lifetime use) on both behavioral and neural group effects reported. Any significant effects of these variables are reported in the Results section.

\section{Behavioral performance}

\section{Reaction times and behavioral adjustment}

$\mathrm{CS}$ and OSU did not differ in average go RTs (mean CS $=659 \mathrm{~ms}$; mean OSU $=639 \mathrm{~ms}, p=0.49)$ or SSRT (mean CS $=187 \mathrm{~ms}$; mean OSU $=187 \mathrm{~ms}, p=0.95)$

As expected (Shenoy and Yu, 2011; Shenoy et al., 2011; Ide et al., 2013), we found a significant positive correlation between go RT and model-based $P($ stop) value individually $(p<0.05)$ in all 

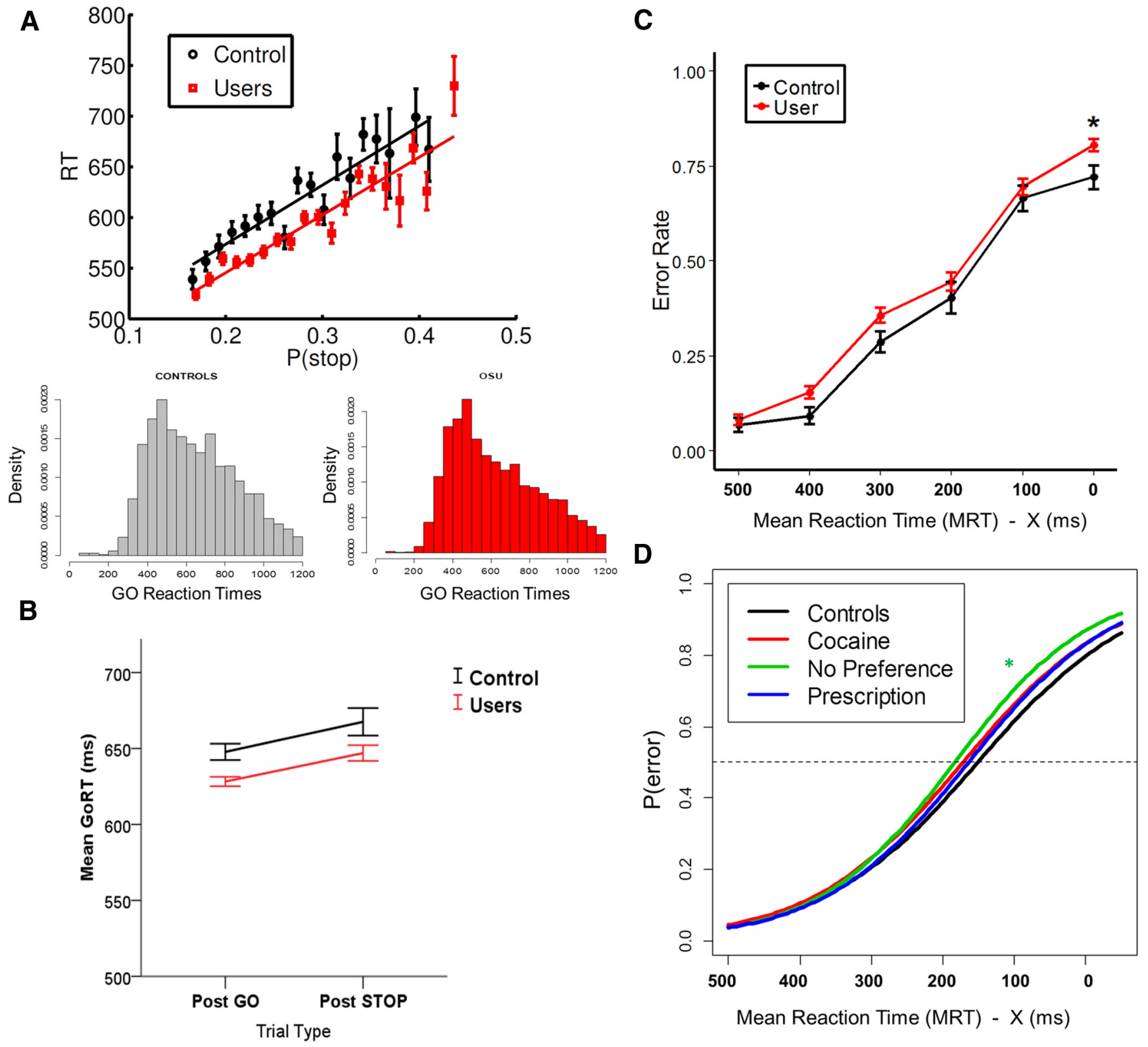

Figure 1. Bayesian model prediction and behavioral performance. A, Bayesian model prediction and behavioral data presented for each group: red/square for OSU, black/circles for CS. As predicted by Bayes-optimal decision making in the stop signal task, participants' go RTs were positively correlated with P(stop) model estimates on each trial. Black (CS) and red (OSU) model lines represent best linear regression fit to mean go RT. Error bars indicate SEM (CS: $n=47 ; 0 S U: n=158) ; B$, Go mean RTs on trials after Go or Stop trials. OSU and CS demonstrate similar slowing after a Stop relative to Go trial. C, Error rates by SSD category for each group. Error bars indicate SEM. D, Fitted logistic inhibitory functions by group (controls: $n=47 ; 0$ SU/Cocaine: $n=47$; OSU/Prescription: $n=57$; OSU/No Preference: $n=54$ ).

subjects (both OSU and CS), with a mean correlation coefficient of $r=0.15$. To assess for any group difference in the linear relationship between RT and $P$ (stop), we applied a generalized linear mixed model (with random intercept and slope) to trial-level RT data with trialwise $P$ (stop) and group modeled as fixed effects and subject modeled as random effect. A strong positive linear relationship between RT and $P$ (stop) was observed $(B=253 \mathrm{~ms}, t=$ $6.9, p<0.001$, model omnibus test: $\left.\chi^{2}=899, p<2.2 \mathrm{e}-16\right)$. Importantly, neither the group main effect $\left(\chi^{2}=0.37, p=0.54\right)$ nor the group $\times P($ stop $)$ interaction $\left(\chi^{2}=0.15, p=0.69\right)$ were significant. Within each group, a strong positive linear relationship was observed (CS: $B=219, p<0.001$; OSU: $B=253, p<$ 0.001 ). Figure $1 A$ shows data collapsed across all subjects for OSU and CS separately, where Go trials were binned by $P($ stop) and average RT was calculated for each bin separately. The figure shows a strong linear relationship between go RT and $P$ (stop).

To investigate poststop slowing and any related group difference, we classified Go trials into two categories based on the preceding type of trial (i.e., go vs stop: successful or failed). As expected, a generalized linear model revealed a strong effect of trial type, reaction times being slower following a stop relative to go trial $(p<0.001)$. However, CS and OSU also did not differ in this behavioral adjustment ( $p=0.68$; Fig. $1 B)$.

Performance accuracy

CS and OSU did not differ in average stop error rates (mean CS = 0.37 ; mean OSU $=0.42, p=0.14$ ). To examine the relationship between group and SSD on error probability, we fitted a general- 
A
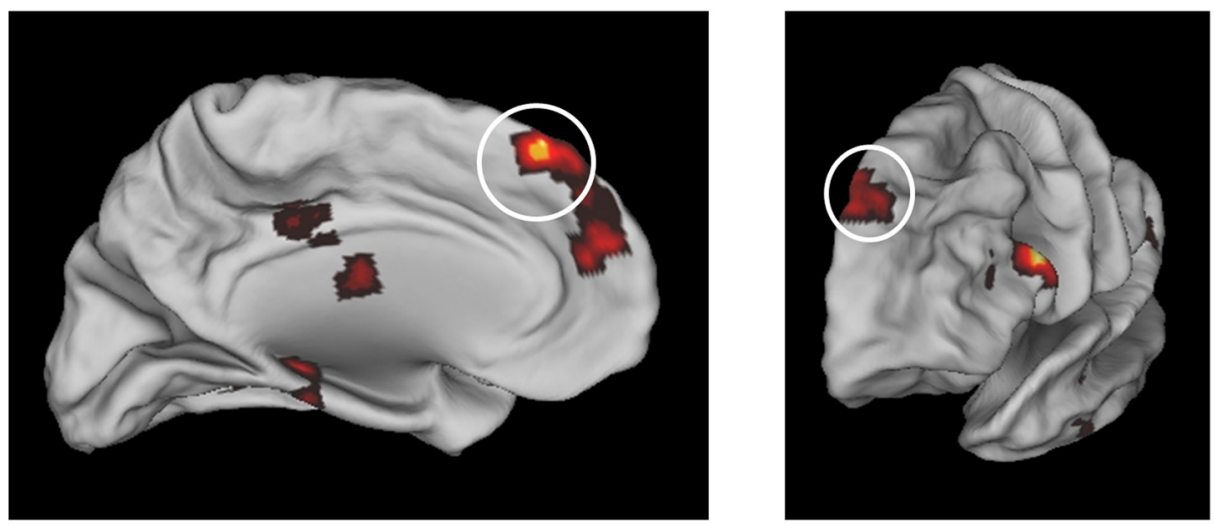

B

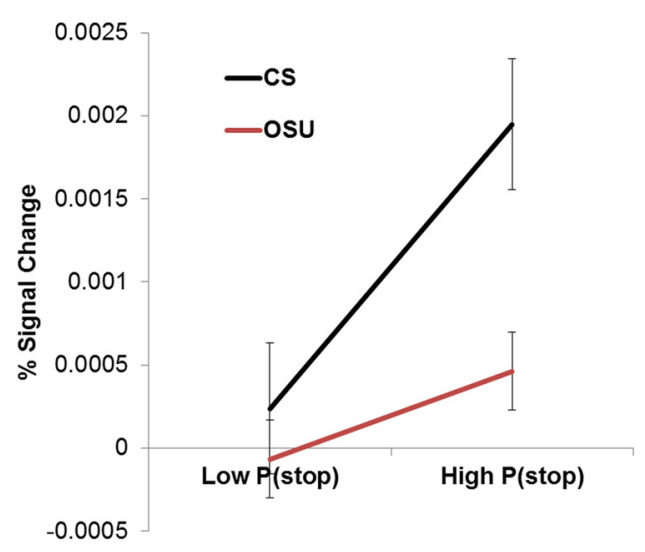

C

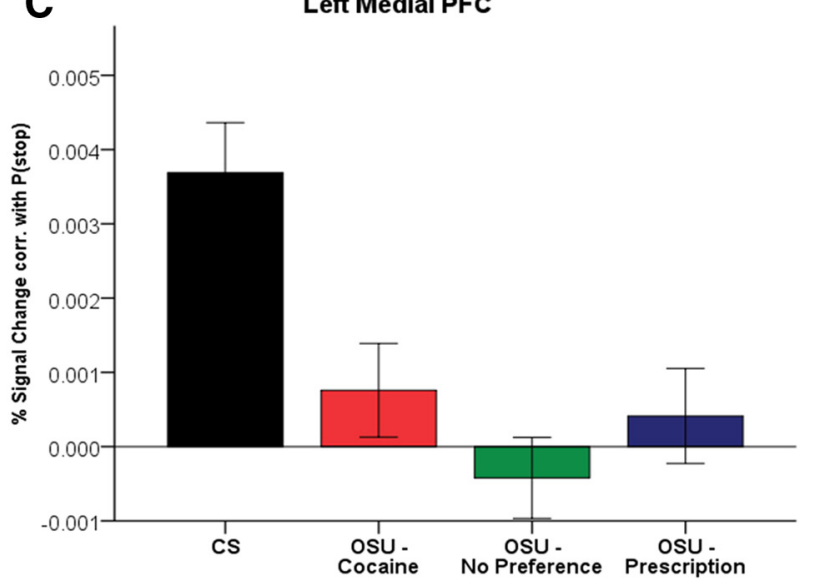

Figure 2. Model-based fMRI data analysis: coding of $P$ (stop) magnitude in the left medial PFC. $A$, Group main effect for BOLD signal associated with $P$ (stop) magnitude (red clusters), including in the left medial PFC (circled). B, Percent signal change on trials with low versus high $P$ (stop) values (based on median split). $\boldsymbol{C}$, Beta coefficient for linear relationship between percent signal change and the parametric regressor $P$ (stop), presented by group (CS: $n=47 ; 0$ SU/Cocaine: $n=47 ; 0$ SU/Prescription: $n=57 ;$ OSU/No Preference: $n=54$ ). Error bars indicate \pm 1 SEM relative to CS. OSU (as a whole) failed to show significant $P($ stop)-dependent activation (Cohen $d=0.79$ ). Beta was not statistically different from 0 in any 0 SU subgroup.

ized mixed linear model with a logistic link function to binary error data, with SSD and group modeled as fixed effects, and subject modeled as random effect. A significant SSD by Group interaction was revealed ( $p=0.02$ ), with OSU having a higher likelihood of error for longer SSDs, as shown in the group average error rates (Fig. 1C). A more specific analysis with drug preference showed this effect was more apparent among OSU in the "no preference" group $(p=0.002)$ and less so in the "prescription" $(p=0.07)$ and "cocaine" $(p=0.10)$ groups (see fitted logistic functions by drug preference, Fig. 1D).

To assess the relationship between $P$ (stop) and error likelihood, we fitted a generalized mixed linear model with a logistic link function to binary error data with trialwise $P$ (stop) and group modeled as fixed effects and subject modeled as random effect. As expected (Shenoy and Yu, 2011; Shenoy et al., 2011; Ide et al., 2013), we found a negative relationship between error likelihood and $P$ (stop), with higher $P$ (stop) prompting a smaller likelihood of error (odds ratio $=0.31$, Wald $z=-3.14, p=$ 0.0016; omnibus test: $\left.\chi^{2}=9.78, p<0.005\right)$. The main effect of group $\left(\chi^{2}=2.6, p=0.11\right)$ and group $\times P($ stop $)$ interaction $\left(\chi^{2}=0.48, p=0.49\right)$ did not reach statistical significance, again suggesting that CS and OSU may similarly rely on an estimate of trial-trial fluctuations in $P$ (stop) to prevent commission errors.

Overall, these results are consistent with our earlier findings (Ide et al., 2013) and suggest that both CS and OSU individuals maintain and use an internal estimate of stop trial probability to make anticipatory adjustments for inhibitory control based on trial history, with systematic consequences in go RT and stop error rate. Broadly speaking, by slowing down as the expectation of stop trial increases, the subject can minimize the risk of a stop error (Shenoy and Yu, 2011).

\section{fMRI analyses}

Based on the above findings, in the GLM, we separately modeled go, stop success, and stop error trials using $P$ (stop) as a parametric modulator (orthogonalized with respect to categorical Go and Stop regressors).

Bayesian prediction of inhibitory response (group comparison) The first comparison of interest involved assessing for any group differences in recruiting brain areas encoding prior $P$ (stop) after regressing out any variance correlated with actual stimulus outcome (stop vs go). Significantly stronger activations (whole brain analysis, corrected for clusterwise significance: $p<0.01)$ in CS relative to OSU were revealed in several areas, including the bilateral medial prefrontal cortex (Brodmann area [BA] 9/10; peak voxel at Talairach coordinate $[\mathrm{TC}]: 0,56,16)$, left medial prefrontal cortex (BA 8; TC: $-4,50,39$; Fig. 2), left caudate (TC: $-22,5,11$ ), right insula (TC: $25,2,5)$ left parahippocampal gyrus (BA 36, TC: $-25,-41,-9$ ), left posterior insula (BA 13, TC: $-38,-32,18$ ), and the left inferior frontal gyrus (BA 9, TC: -50 , 20, 21; Fig. 3A).

To quantify the relationship between occasional stimulant use and activity in these brain areas, we used Huber regressions to identify any subregions in which $P$ (stop) activation may be predicted by 
A

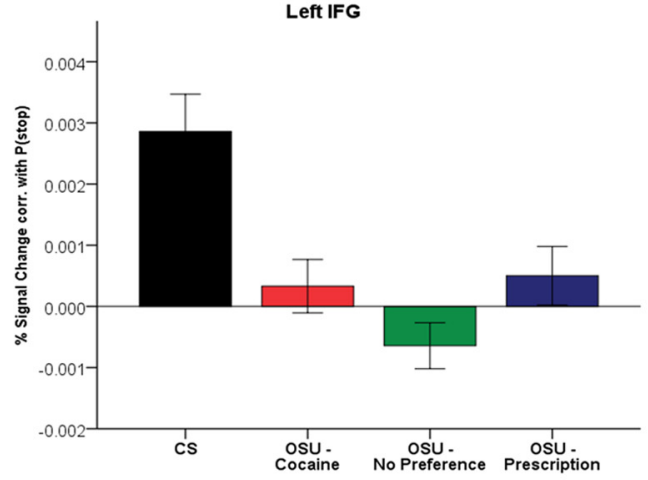

B

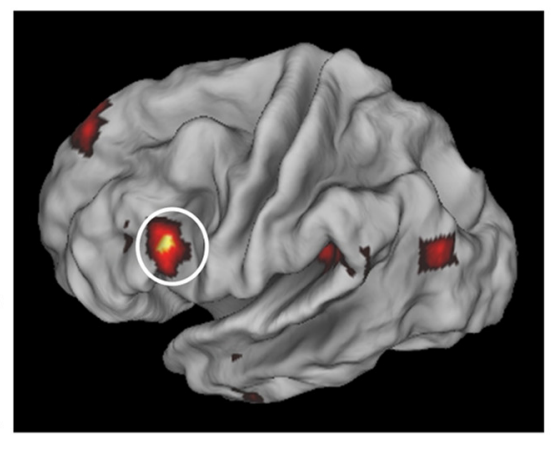

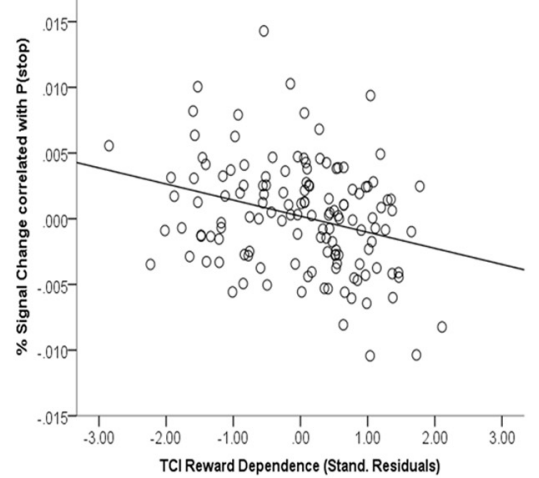

Figure 3. Model-based fMRI data analysis: coding of $P($ stop) magnitude in the left IFG. $A$, Group main effect for BOLD signal associated with $P($ stop) magnitude in the left IFG. Bar graph shows the average percent signal change correlated with $P($ stop) for each group (error bars indicate \pm 1 SEM). Relative to controls (CS), OSU failed to show significant $P$ (stop)-dependent activation (Cohen $d=0.72$ ). Beta was not statistically different from 0 in any OSU subgroup. $\boldsymbol{B}, P($ stop)-modulated activation in this region was negatively correlated $(r=-0.28, p<0.05)$ with the TCl reward dependence scale in OSU (scatterplot).
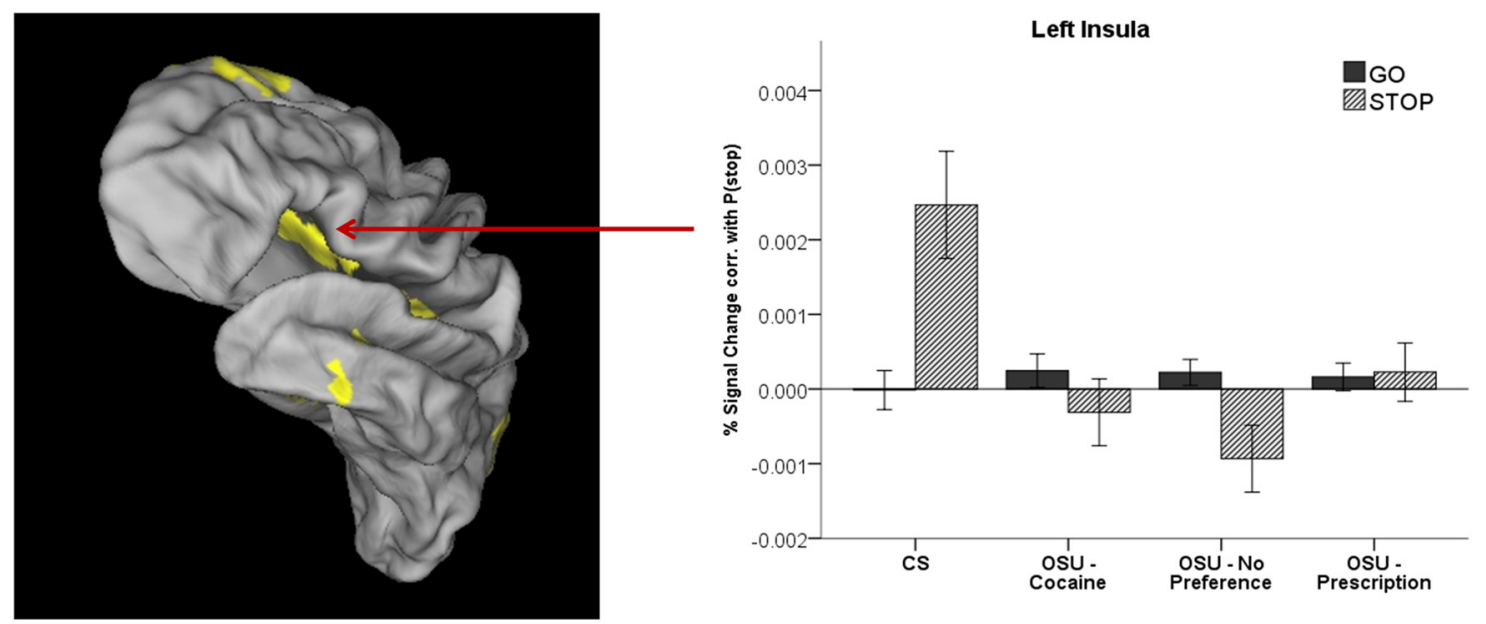

Regions encoding significant interaction in $\mathrm{P}(\mathrm{stop})$ modulation between trial type and group

Figure 4. Left, BOLD signal in the left insula and other regions showing a significant interaction of group (CS vs OSU) and trial type (Go vs Stop) on P(stop) modulation. Right, Bar graph represent average $P$ (stop) modulation of percent signal change by trial type (Go vs Stop) and group [error bars indicate \pm 1 SEM; Controls/CS: $n=47 ; 0$ SU/Cocaine: $n=47 ; 0$ SU/Prescription: $n=57 ; 0$ SU/No Preference: $n=54$; relative to $[S]$. OSU showed smaller or nonsignificant $P($ stop) modulations on Stop trials (Cohen $d=0.67$ ), whereas neither group showed a significant $P($ stop) modulation on Go trials.

clinical measures within OSU. Although none of the reported drug use measures correlated with average $P$ (stop) activation, activation from the left IFG was negatively correlated with TCI reward dependence scores $(r=-0.28, p<0.05$; area significant in voxelwise Huber regression, surviving multiple comparison correction for clusterwise significance: $p<0.05$; Cloninger et al., 1994). Therefore, a stronger responsiveness to signals of reward and social approval (higher TCI reward dependence scores) was associated with lower activation in these regions in response to $P$ (stop) (Fig. $3 B$ ). This psychological index, which has been associated with prefrontal hypoactivations (Sugiura et al., 2000) and lower basal noradrenergic activity (Cloninger, 1986), may therefore be useful among OSU to predict future failure to engage cognitive control.

\section{Modulation of Bayesian prediction error by occasional} stimulant use

Next, we investigated neural correlates of the Bayesian prediction error associated with stimulus outcome, that is, activations correlated with the difference between the actual trial type (i.e., 1 for Stop trial, 0 for Go trial) and predicted $P$ (stop). We were particularly interested in how these neural activations might be differ- ent between OSU and CS. We first identified brain areas associated with a significant interaction between group (OSU vs $\mathrm{CS})$ and $P($ stop $)$ modulated trial type [Stop $\times P($ stop $)$ vs Go $\times$ $P($ stop) $]$. Several regions were identified using this group contrast, including bilateral left and right ACC (BA 32, TC: - 1, 25, 30; BA32, TC: 17, 21, 31), left insula (BA 13, TC: $-26,5,12$ ), right insula (BA 13, TC: 28, -3, 14), left superior/medial PFC (BA8, TC: $-6,46,42)$, bilateral medial PFC (B10, TC: 0, 57, 18), left posterior cingulate gyrus (BA23, TC: $0,-55,20)$, right midcingulate gyrus (and BA 31, TC: 15, -23, 42), and left middle temporal gyrus (TC: $-40,-33,12$ ).

In some of those regions (i.e., left insula, left and right posterior cingulate, and left temporal gyrus), CS showed a positive activation associated with $P$ (stop) on Stop trials, but the correlation with $P$ (stop) on Go trials was not significantly different from $0(p>0.05)$. OSU showed no differential activations to go versus Stop trials in these regions (Fig. 4, left insula).

Signed Bayesian prediction error. In other regions (i.e., left superior medial PFC/B9 and bilateral medial PFC/B10; Fig. $5 A$ ), CS showed a positive correlation with $P$ (stop) in both Go 

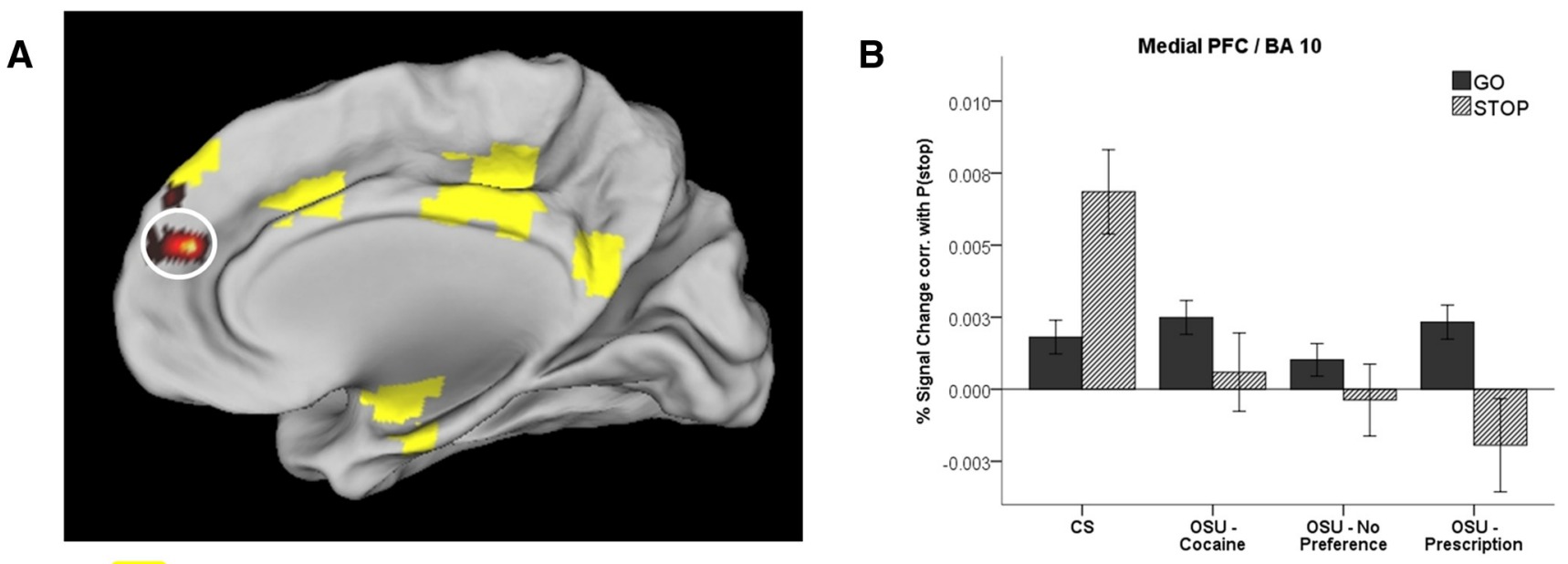

Regions encoding significant interaction between trial type and group.

Regions showing group difference in Bayesian signed prediction error (SPE)

C

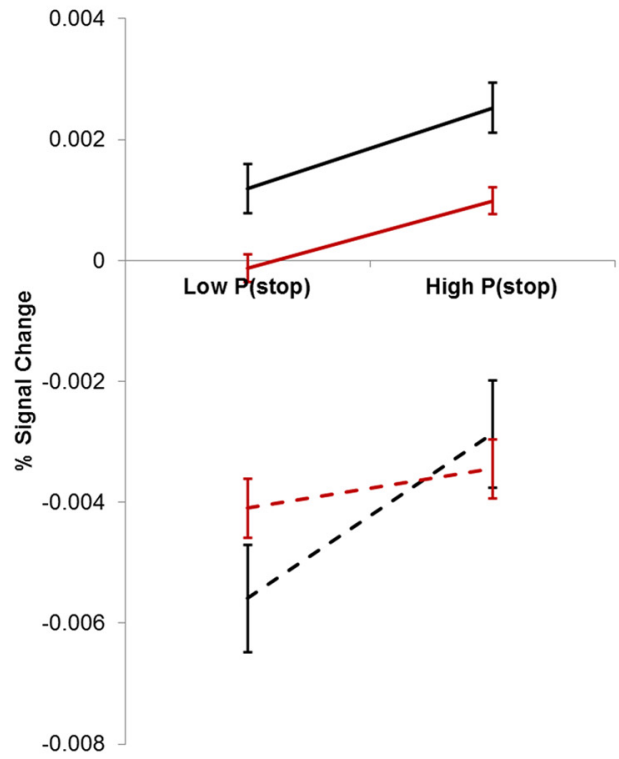

D

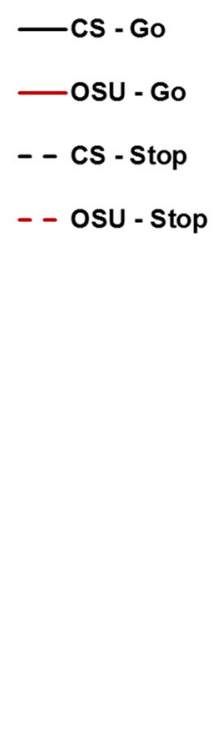

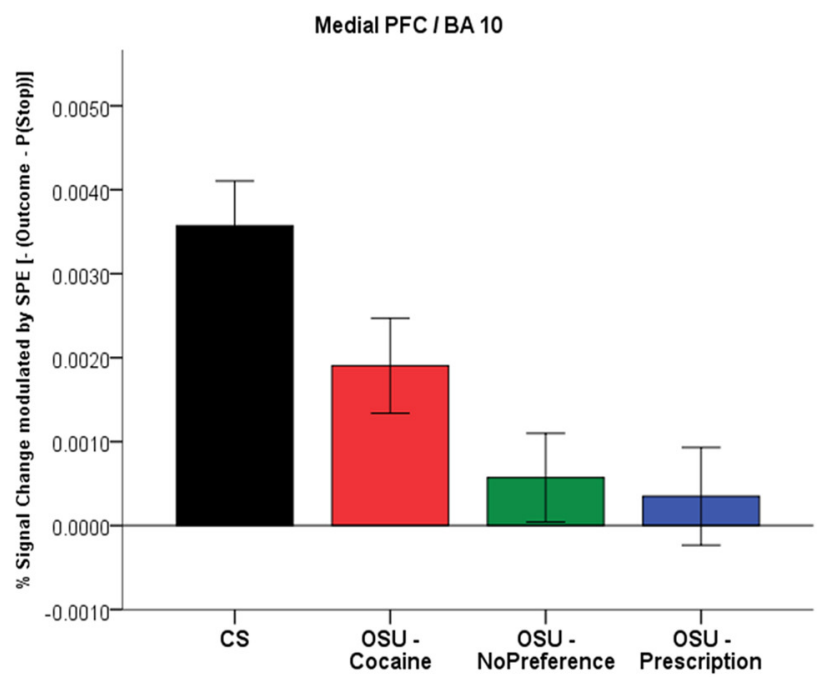

Figure 5. Model-based fMRI data analysis: coding of the Bayesian signed prediction error (SPE) in the medial PFC. $A, B O L D$ signal in the medial PFC (BA 10) showing group difference in percentage signal change modulation by SPE (red cluster). Yellow areas are regions representing a significant interaction between group and $P($ stop)-modulated trial type. $\boldsymbol{B}$. Bar graph displays average $P($ stop) modulation of percent signal change by trial type (Go vs Stop) and group (CS: $n=47 ; 0 \mathrm{SU} /$ Cocaine: $n=47 ; 0$ SU/Prescription: $n=57 ; 0$ SU/No Preference: $n=54$; error bars indicate \pm 1 SEM). In this area, CS demonstrated positive correlations between percentage signal change and $P$ (stop) on both Go and Stop trials, whereas OSU failed to show significant $P$ (stop)-dependent activation (not statistically different from 0 in any OSU subgroup); $C$. Percent signal change on trials with low versus high $P($ stop) values (based on median split); $\boldsymbol{D}$. Average percent signal change correlation with a negative SPE for each group (error bars: \pm 1 SEM). Relative to CS, OSU showed weaker SPE-dependent activation (Cohen $d=0.69$ ). Beta was not statistically different from 0 for the No Preference and Prescription subgroups.

and Stop trials, which is consistent with a negative signed prediction error [i.e., $-($ outcome $-P($ stop $))=P($ stop $)-$ outcome]. This is because $P($ stop $)-$ outcome $=P($ stop $)-0=$ $P$ (stop) on Go trials (since the probability of encountering a Stop trial on a sure Go trial is 0 ) and $=P($ stop $)-1$ on Stop trials (since the probability of encountering a Stop trial on a sure Stop trial is 1). Therefore, the negative SPE is equal to $P$ (stop) or $P($ stop $)-1$ depending on trial type and is therefore positively related to $P$ (stop) for both Go and Stop trials (see Materials and Methods). Unlike CS, OSU failed to show $P$ (stop)-modulated activation on Stop trials. Although they generally showed a positive $P$ (stop) modulation on Go trials, this effect was weaker (Fig. $5 B, C$ ). Results from our BPE parametric analyses confirmed attenuated signed prediction error activations, which was most apparent in the no preference and prescription groups (Fig. 5D).

Unsigned Bayesian prediction error. Finally, in other regions (i.e., left dACC and right insula; Fig. $6 A$ and Fig. 7A), CS showed a positive correlation with $P$ (stop) on Stop trials and a negative correlation with $P$ (stop) on Go trials. This is consistent with activations associated with a negative unsigned prediction error $(-\mid$ outcome $-P($ stop $) \mid)$. This is because an unsigned prediction error $=\mid$ outcome $-P($ stop $)|=| 0-P($ stop $) \mid=P($ stop $)$ on Go trials and $=\mid 1-P($ stop $) \mid=1-P($ stop) on Stop trials (because $P($ stop) values are $>0$ and $<1$ and thus $1-P($ stop $)$ is always $>0$; see Materials and Methods). Again, unlike CS, OSU failed to show a differential activation to Go versus Stop trials in those regions (Figs. 
A

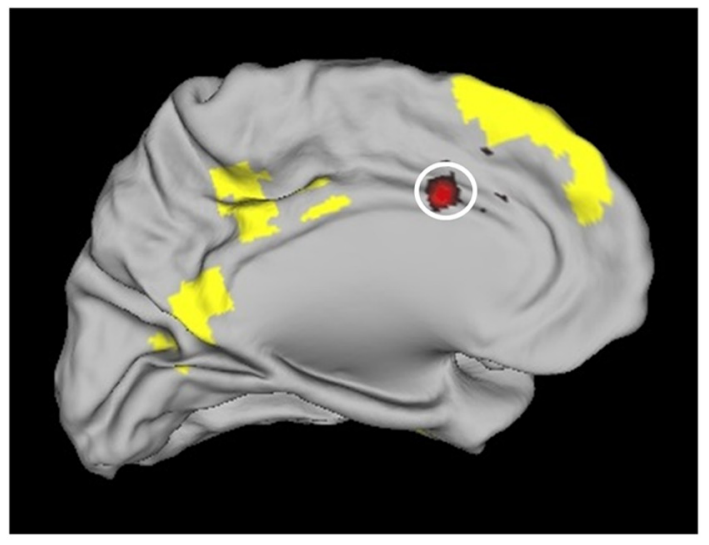

B

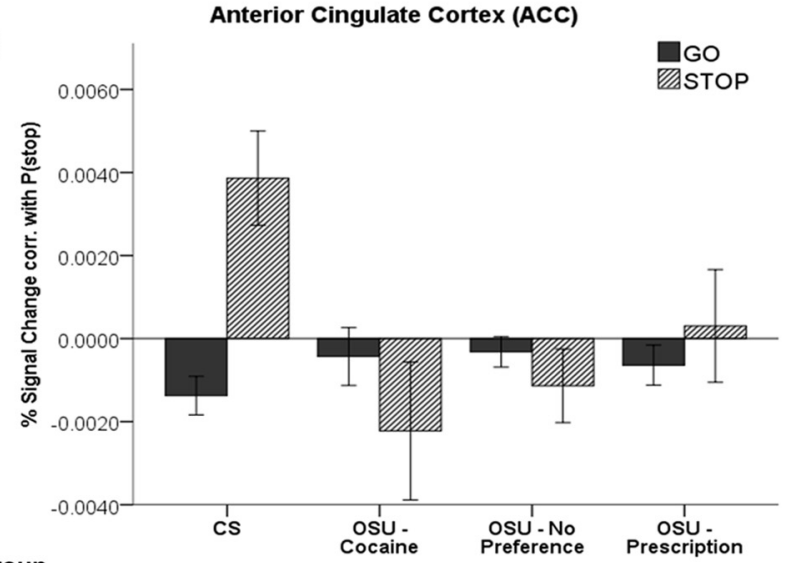

Regions encoding significant interaction between trial type and group.

Regions showing group difference in Bayesian unsigned prediction error (UPE)

C

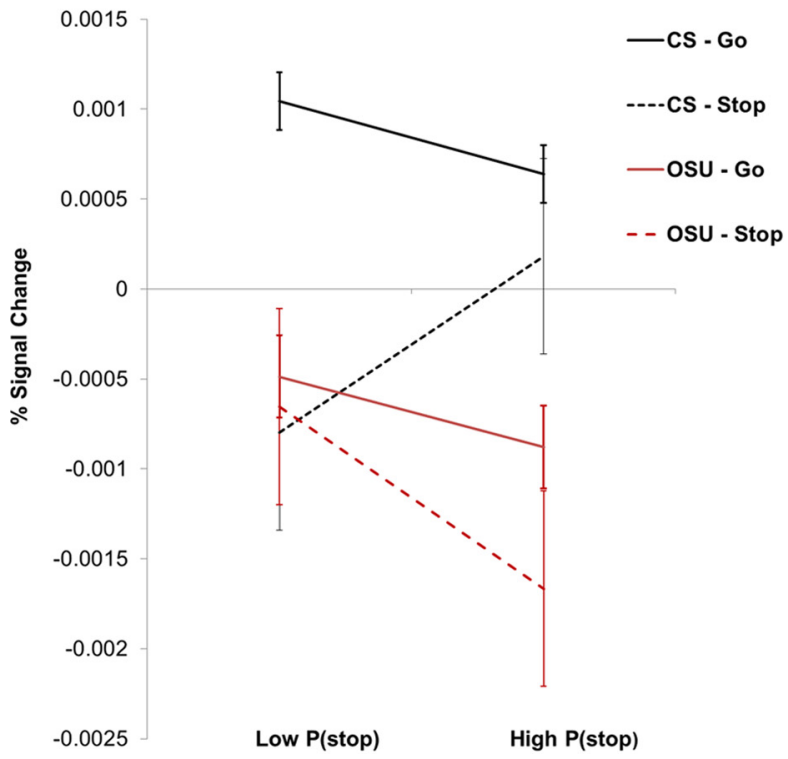

D

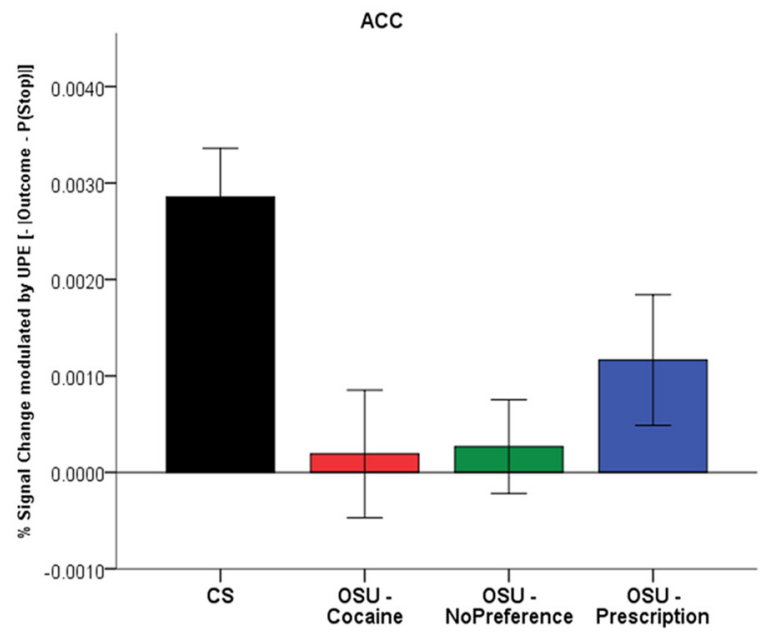

Figure 6. Model-based fMRI data analysis: coding of the Bayesian unsigned prediction error (UPE) in the ACC. $A$, BOLD signal in the ACC (circled) showing group difference in percentage signal change modulation by UPE (red clusters). Yellow areas are regions representing a significant interaction between group and $P($ stop)-modulated trial type. $\boldsymbol{B}$, Bar graph displays average $P($ stop) modulation of percent signal change by trial type (Go vs Stop) and group (CS: $n=47 ; 0$ SU/Cocaine: $n=47 ;$ OSU/Prescription: $n=57 ; 0$ SU/No Preference: $n=54$; error bars indicate \pm 1 SEM). In this area, CS demonstrated a negative correlation between percentage signal change and $P($ stop) on Go trials and a positive correlation on Stop trials, whereas OSU failed to show significant $P($ stop)-dependent activation (not statistically different from 0 in any OSU subgroup). C, Percent signal change on trials with low versus high P(stop) values (based on median split). D, Average percent signal change correlation with a negative UPE for each group (error bars: \pm 1 SEM). Relative to controls (CS), OSU showed weaker UPE-dependent activation (Cohen $d=0.57$ ). Beta was not statistically different from 0 in any OSU subgroup.

$6 B, C, 7 B, C)$. Importantly, based on parametric analyses for UPE, OSU had significantly attenuated UPE activations, which was not statistically different from zero for the cocaine and no preference groups (Figs. 6D, 7D).

No areas revealed any negative activations to $P($ stop) on both Go and Stop trials (which would reflect group difference in activation associated with a signed prediction error). In follow-up analyses, Huber regressions revealed no correlation between neural activation in these areas and drug use measures or clinical self-report measures.

$\mathrm{P}($ stop) modulation of inhibitory success (group difference) To assess whether stimulant use may further affect the degree to which performance accuracy further modulates neural activations correlated with $P$ (stop), we searched for areas associated a significant interaction between group and $P($ stop)-modulated activation for SS versus SE trials. Only one region in the right parahippocampal gyrus (BA 28, TC: 27, - 12,-13) was identified for this contrast. Specifically, CS had a stronger positive correlation to $P$ (stop) on SE trials relative to SS. In contrast, OSU showed no differential activation.

\section{Bayesian prediction of inhibitory response (ROIs)}

Given prior evidence suggesting that the IFG and STN play a key role in the implementation of response inhibition in the stop signal task (Aron et al., 2004), we assessed for trial-type effects in these ROIs. In the right IFG, we found a significant positive correlation with $P$ (stop) for Stop trials in both groups, whereas $P($ stop)-modulated activation was not significantly different from 0 for Go trials in either group. However, OSU exhibited significantly weaker activation on 
A

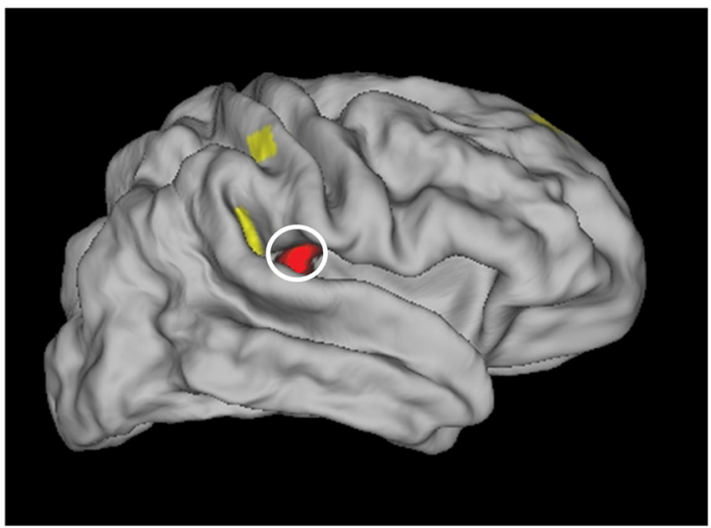

Regions encoding significant interaction between trial type and group.
B

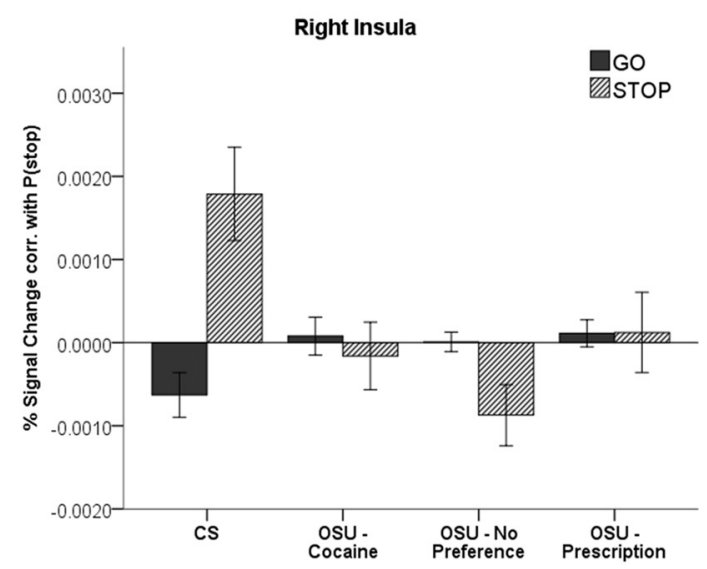

Regions showing group difference in Bayesian unsigned prediction error (UPE)

C

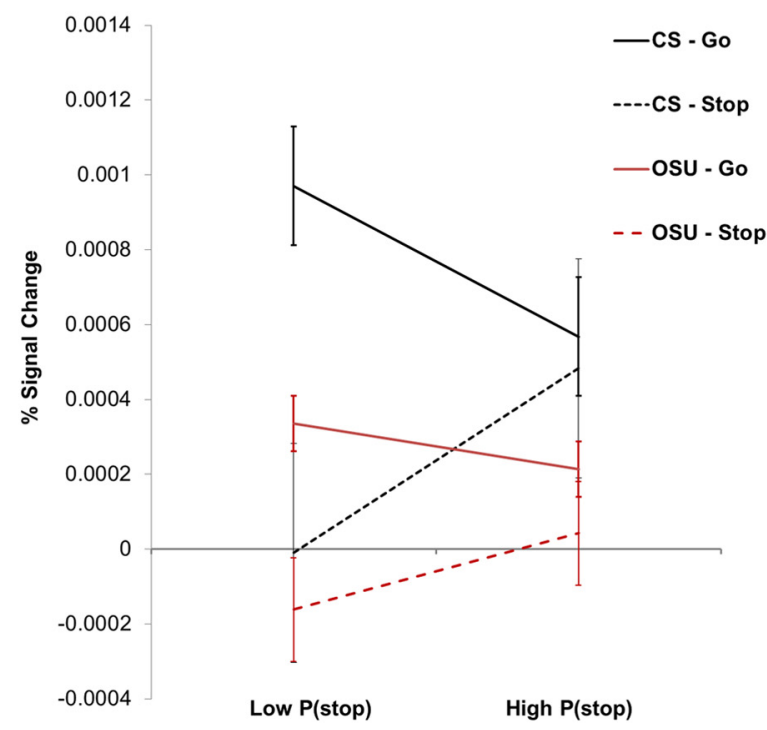

D

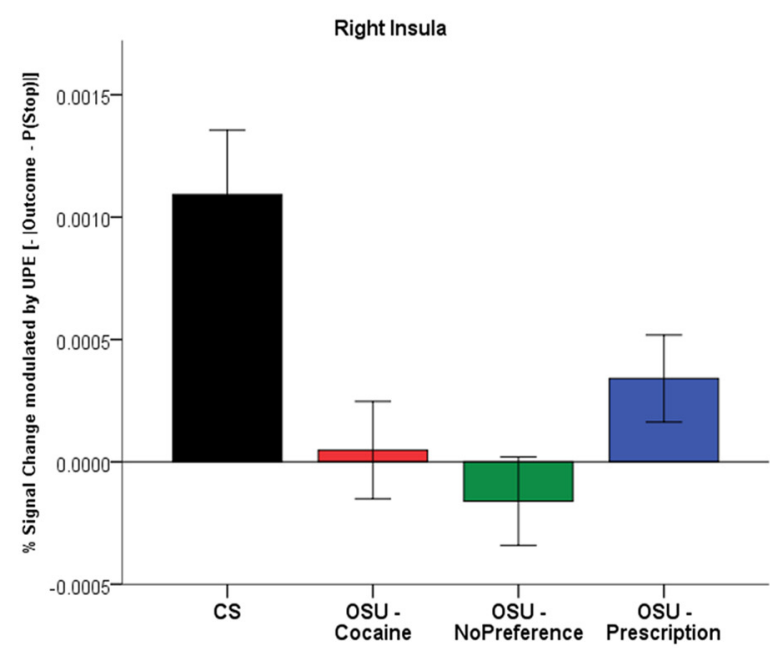

Figure 7. Model-based fMRI data analysis: coding of the Bayesian unsigned prediction error (UPE) in the right insula. $A$, BOLD signal in the right insula (circled) showing group difference in percentage signal change modulation by UPE (red clusters). Yellow areas are regions representing a significant interaction between group and $P($ stop)-modulated trial type. $\boldsymbol{B}$, Bar graph displaying average $P$ (stop) modulation of percent signal change by trial type (Go vs Stop) and group (CS: $n=47 ; 0 S U / C o c a i n e: ~ n=47 ; 0 S U / P r e s c r i p t i o n: n=57 ; 0 S U /$ No Preference: $n=54 ;$ error bars indicate \pm 1 SEM). In this area, CS demonstrated a negative correlation between percentage signal change and $P($ stop) on Go trials and a positive correlation on Stop trials, whereas OSU failed to show significant $P$ (stop)-dependent activation (not statistically different from 0 in any 0 SU subgroup). C, Percent signal change on trials with low versus high $P($ stop) values (based on median split). $\boldsymbol{D}$, Average percent signal change correlation with a negative UPE for each group (error bars: \pm 1 SEM). Relative to CS, OSU showed weaker UPE-dependent activation (Cohen $d=0.63$ ). Beta was not statistically different from 0 in any of the cocaine and no preference subgroups (and marginally significant for the prescription group: $p=0.06$ ).

SS trials relative to CS. We further found that this effect was driven by the "No preference" ( $p=0.003)$ and "Cocaine" $(p=0.04)$ groups, but was not apparent in the "Prescription" OSU group (Fig. 8A, IFG activations). In the right and left STN, both groups showed a stronger correlation to $P$ (stop) on SS trials relative to both Go and SE trials. No group difference was observed for any trial type (Fig. $8 B$ ). These results are consistent with the notion that IFG and STN regions both play critical roles in the anticipation of stop signals and the implementation of a stop response with STN activation further modulated by performance success.

\section{Group comparisons for categorical regressors}

We conducted similar LME analyses on the categorical regressors (Go, SS, SE) to assess any group differences in activation related to trial type after regressing out any variance correlated with the $P$ (stop)-modulated regressors. Because the parametric regressors are orthogonalized to the categorical predictors in our GLM, this analytical approach thus allowed us to distinguish neural activations involved in detecting and responding to stop signals and successful inhibition [above and beyond $P$ (stop)-modulated activations].

Go versus Stop. For the group overall main effect (Stop $+\mathrm{Go}$ ), significantly stronger activations were observed in CS relative to OSU in the right superior temporal gyrus (BA: 22/TC: $48,-19$, $-6)$, left posterior cingulate gyrus (BA31, TC: $-15,-39,22$ ), and the right parahippocampal gyrus (BA 27/TC: 22, -30, -6). In addition, a significant group by trial type interaction was observed in the left superior parietal lobule (BA 7, TC: $-33,-51$, 51 ), right midcingulate gyrus (BA 24 , TC: $11,-18,42$ ), and right postcentral gyrus (BA 3, TC: $30,-23,45$ ). In all three areas, CS showed significantly more deactivation to stop relative to Go trials. In contrast, OSU demonstrated attenuated differential activations compared with CS in the cingulate and postcentral 
A

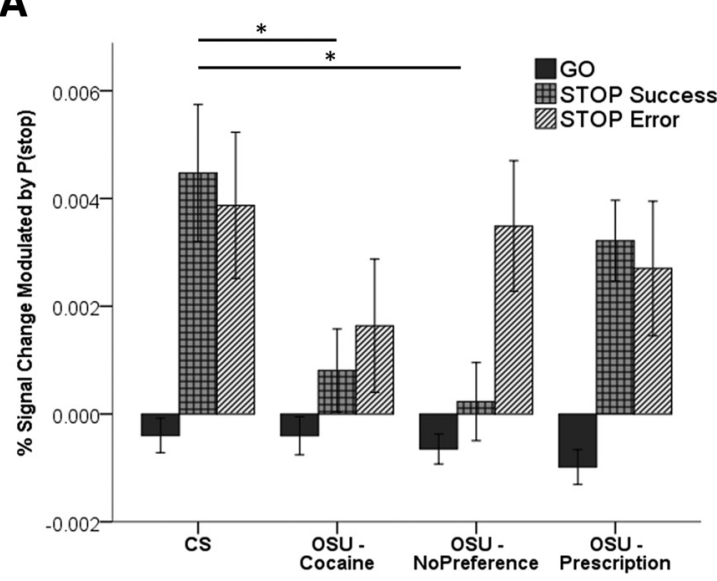

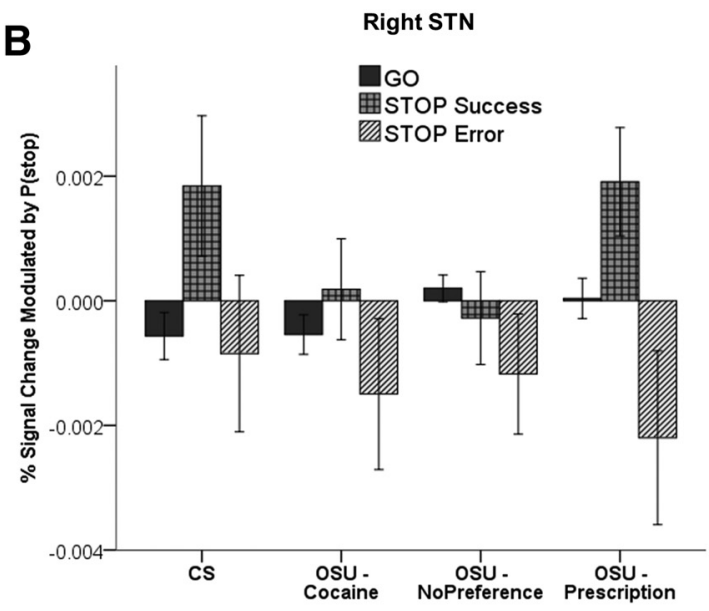

Figure 8. Trial-outcome-dependent $P($ stop) activation for anatomical ROIs in the IFG $(\boldsymbol{A})$ and right STN $(\boldsymbol{B})$. Bar graphs represent average percent signal change for parametric regressors Go $\times P($ stop), SE $\times P($ stop), and SS $\times P($ stop $)$ in CS $(n=47)$ and OSU (OSU/Cocaine: $n=47$; OSU/Prescription: $n=57 ;$ OSU/No Preference: $n=54)$. Relative to CS, OSU in the Cocaine and No Preference groups had significantly lower $P($ stop)-modulated activation on stop success trials; no group difference was observed in the right STN. Error bars indicate \pm 1 SEM.

gyrus and no differential activation to Stop vs Go trials in the parietal lobule. Although there is some degree of functional overlap (e.g., mid/posterior cingulate) with areas implicated for $P$ (stop)-modulated activation, these findings could reflect a weaker recruitment in OSU of a network more specifically involved in the detection and processing of Stop (vs Go) trials above and beyond group differences in regions participating in the anticipation of a stop signal.

Stop success versus Stop error. When examining differences in success of inhibition implementation (success vs error), we identified a significant interaction between group and stop trial type (SS vs SE) in two brain areas: the right precuneus (BA 7, TC: 17, $-63,48$ ) and the right IFG (BA 46, TC: $43,39,5$ ). In these areas, CS showed stronger activation on SS relative to SE trials, whereas OSU showing the opposite pattern (i.e., stronger activation on SE trials), which is consistent with a deficit among OSU in recruiting these regions when processing Stop versus Go trials independently of stop trial anticipation processes.

ROI analyses. Finally, the ROI analyses in the right IFG showed that CS demonstrated stronger activations in this area on SS trials relative to SE trials, whereas this difference was not significant in OSU. In the STN, a pattern similar to those associated with $P$ (stop)-modulated regressors (and no group difference) was observed. Specifically, both groups showed a stronger activation to SS trials relative to SE trials. These results again suggest that both IFG and STN play an important role in detecting and responding to the stop signal.

\section{Discussion}

We applied a Bayesian ideal observer model to investigate differences in probabilistic expectations of inhibitory response between OSU and CS. fMRI was used to assess the neural basis of proactive control in stopping in a stop-signal paradigm. To our knowledge, this is the first neural investigation using a Bayesian model of inhibitory function in OSU, a population at higher risk for developing dependence. OSU showed reduced brain activations associated with trial-level expectation of inhibitory needs (i.e., $P($ stop)) in the medial prefrontal cortex, caudate, and left IFG. OSU also had weaker Bayesian prediction error signals (representing the discrepancy between predicted probability of stop trial and actual stimulus outcome) in the medial PFC, dACC, and right insula.

\section{Behavioral performance.}

Consistent with the minimal behavioral differences often observed in this population (Colzato et al., 2007; Reske et al., 2011), OSU demonstrated only subtle alterations in inhibitory performance, mostly in the form of greater impulsiveness in trading off accuracy for speed: OSU showed a trend toward faster go reaction time and an OSU subgroup (using both prescription stimulants and cocaine) made more commission errors for longer stop signal delays. However, group model fits suggest that they did not significantly differ from CS in adjusting their reaction times with respect to anticipated $P$ (stop).

\section{Bayesian prediction of inhibitory response in OSU}

OSU had attenuated neural activations associated with overall $P$ (stop) magnitude in several areas, including the left medial PFC, left caudate, and left IFG. OSU appeared to engage the frontostriatal network less when computing dynamic probabilistic state estimations in inhibitory control. The attenuation of the medial PFC in OSU is consistent with previous results in methamphetamineand cocaine-dependent individuals, who showed weaker medial prefrontal activations during decision making (Bolla et al., 2003; Paulus et al., 2003). This is also consistent with previous suggestions that the medial PFC participates in the encoding of dynamic Bayesian estimates of internal state/position, predicting RT adjustment and decision making (Yoshida and Ishii, 2006). In addition, the dorsal part of the caudate (as found here) has strong functional connectivity to various prefrontal and parietal areas (Di Martino et al., 2008) and is implicated generally in cognitive control and specifically in learning stimulus-action associations (White, 1997; O’Doherty, 2004).

\section{Modulation of Bayesian prediction error by occasional stimulant use}

OSU demonstrated attenuated Bayesian prediction error (PE) signals in several areas, including the dACC and insula (for unsigned $\mathrm{PE}$ ) and medial PFC (for signed PE), suggesting a weakened repre- 
sentation of the unexpectedness of surprising outcomes. Specifically, OSU showed attenuated dACC responses correlating with a negative UPE (i.e., $-\mid$ outcome $-P$ (stop) $\mid$ ), which is consistent with previous findings in neural recording studies of a negative relationship between dACC activity and prediction error (Seo and Lee, 2007; Hayden et al., 2011; Kennerley et al., 2011). The results here are also broadly consistent with our recent finding that dACC encodes a Bayesian prediction error related to $P$ (stop) in the stop-signal task (Ide et al., 2013), as well as earlier work implicating ACC in the encoding of various types of expectancy violations in animals (Kennerley et al., 2011) and humans (Somerville et al., 2006; Aarts and Roelofs, 2011), although in those earlier works any correlation of ACC with UPE was positive rather than negative. We note that although our previous work identified a positive correlation between dACC activation and UPE (Ide et al., 2013), the region identified there was more dorsal than that identified here.

Error prediction/expectancy violation signals have also been observed in the insula in the context of reinforcement learning (Murray et al., 2008; Preuschoff et al., 2008; Bossaerts, 2010). The insula is thought to support the representation of current and predictive feeling states, allowing for error-based learning in complex, dynamic environments (Singer et al., 2009). Some computational investigations have also linked activation of both the dACC (Behrens et al., 2007; Rushworth and Behrens, 2008) and the insula (Preuschoff et al., 2008; Singer et al., 2009; Bossaerts, 2010) to the coding of surprise and uncertainty/risk in the environment (i.e., volatility). The group differences observed in these areas could therefore relate to weaker neural responses to the surprise or uncertainty associated with stimulus outcome.

Reduced ACC recruitment in the present study is consistent with previous neural investigations showing ACC hypoactivations in stimulant users in the context of inhibitory control and decision-making paradigms (Bolla et al., 2004; Hester and Garavan, 2004; Goldstein and Volkow, 2011). Similarly, recent studies have found attenuated insula responses in stimulant users (Paulus et al., 2005; Clark et al., 2012), including nondependent individuals (Paulus et al., 2008; Stewart et al., 2013). This research suggests that stimulant users deploy less neural resources for trend detection and prediction, which is associated with difficulties adapting to changing task contexts (Verdejo-García et al., 2005; Aron and Paulus, 2007). Importantly, these neural markers have been shown to predict relapse (Paulus et al., 2005; Clark et al., 2012). Therefore, the present findings suggest that, beyond altered speed/accuracy tradeoff and related impulsivity, OSU may exhibit behavioral rigidity and reduced ability to adjust their inhibitory behavior in dynamic environments, which may in turn contribute to difficulties maintaining abstinence (e.g., failing to increase internal estimations of the need to say "no" following an increase in peer pressure).

Interestingly, the ACC and insula have structural (Ridderinkhof et al., 2004; Cauda et al., 2011) and functional (Margulies et al., 2007; Zago et al., 2008) reciprocal connections with the medial PFC, IFG, and caudate, which were involved in $P$ (stop) magnitude coding in the present study. This is consistent with a role of the ACC in tracking the accuracy of the $P($ stop) belief model with UPE signals and in mediating the updating of these probabilities (O'Reilly et al., 2013) represented within an executive frontostriatal network. In contrast, SPE group differences were observed in the superior medial PFC (BA 9/10), regions adjacent and functionally connected to the posterior mPFC/BA 8 (Arikuni et al., 1988) and IFG (Kemmotsu et al., 2005), which showed activation correlations with $P$ (stop) and premotor areas (Euston et al., 2012). These superior mPFC regions may thus play a role in modulating response selection based on directional PEs, a network that appears to be impaired in OSU.

\section{Bayesian prediction and implementation of inhibitory control}

Neural evidence from both primate (Schall et al., 2002; Paré and Hanes, 2003; Stuphorn and Schall, 2006) and human (Aron et al., 2003; Aron and Poldrack, 2006) studies have pointed to the right IFG and STN as important for implementing the stop action that terminates or cancels the go response in a competitive race model (Logan and Cowan, 1984). Stronger recruitment of these areas has been linked to more successful stopping and shorter SSRTs. Our results suggest that activity in these areas are indeed modulated by $P$ (stop) on all Stop trials in the IFG and successful stops in the STN. This computational anticipatory process may therefore modulate the recruitment of regions involved in implementing the stop process. Although, for the most part, OSU and CS showed similar patterns of activation in this area, OSU demonstrated weaker activations of the right IFG on successful Stop trials, suggesting subtle deficits in updating the probability of a stop signal to adjust behavior. Interestingly, OSU using both cocaine and prescription stimulants showed higher error tendency and weakest $P$ (stop)-modulated activation in the right IFG, suggesting that these individuals may be at higher risk for developing inhibitory control deficits.

\section{Summary}

We have shown here that OSU use less brain-processing resources in the dACC, medial PFC, and insula to predict and update the need for response inhibition, processes that are critical for adjusting and optimizing behavioral performance. Based on evidence that OSU and CS had similar Bayesian model accounts of trial-by-trial variability in go RT and stop error rate, the lower correlation coefficients in these brain regions among OSU are unlikely to reflect differential adequacy of model fit. Moreover, as we argued previously (Yu and Dayan, 2005), the ACC is likely to cooperate with the noradrenergic system to track unexpected uncertainty (e.g., large, uncommon changes in environmental contingencies) beyond expected, task-related uncertainty (e.g., trial-to-trial fluctuations of predictive relationships). Therefore, we suggest that the weaker $P$ (stop) prediction error signals in OSU may reflect group differences in tracking unexpected, large changes (e.g., drastic changes in stop signal frequency or temporal onset distribution) in the task, rather than in fine-tuning $P$ (stop) estimation on a trialwise basis. Such neural alterations could lead to behavioral deficits in more complex and uncertain environments. Because the current task did not incorporate an element of unexpected uncertainty, it is perhaps unsurprising that behavioral tracking of $P$ (stop) did not appear to be impaired in OSU despite an attenuated dACC UPE signal. Given the relatively recent onset and low level of stimulant use in this population, future research involving more complex tasks is needed to determine the precise function of the dACC UPE signal and related behavioral impairments in simulant-dependent individuals and any predictive relationship to relapse.

\section{References}

Aarts E, Roelofs A (2011) Attentional control in anterior cingulate cortex based on probabilistic cueing. J Cogn Neurosci 23:716-727. CrossRef Medline

Arikuni T, Watanabe K, Kubota K (1988) Connections of area 8 with area 6 in the brain of the macaque monkey. J Comp Neurol 277:21-40. CrossRef Medline 
Aron AR, Poldrack RA (2006) Cortical and subcortical contributions to stop signal response inhibition: role of the subthalamic nucleus. J Neurosci 26:2424-2433. CrossRef Medline

Aron AR, Fletcher PC, Bullmore ET, Sahakian BJ, Robbins TW (2003) Stopsignal inhibition disrupted by damage to right inferior frontal gyrus in humans. Nat Neurosci 6:115-116. CrossRef Medline

Aron AR, Robbins TW, Poldrack RA (2004) Inhibition and the right inferior frontal cortex. Trends Cogn Sci 8:170-177. CrossRef Medline

Aron JL, Paulus MP (2007) Location, location: using functional magnetic resonance imaging to pinpoint brain differences relevant to stimulant use. Addiction 102:33-43. CrossRef Medline

Barratt ES (1959) Anxiety and impulsiveness related to psychomotor efficiency. Perceptual and Motor Skills 9:191-198. CrossRef

Behrens TE, Woolrich MW, Walton ME, Rushworth MF (2007) Learning the value of information in an uncertain world. Nat Neurosci 10:12141221. CrossRef Medline

Bolla KI, Eldreth DA, London ED, Kiehl KA, Mouratidis M, Contoreggi C, Matochik JA, Kurian V, Cadet JL, Kimes AS, Funderburk FR, Ernst M (2003) Orbitofrontal cortex dysfunction in abstinent cocaine abusers performing a decision-making task. Neuroimage 19:1085-1094. CrossRef Medline

Bolla K, Ernst M, Kiehl K, Mouratidis M, Eldreth D, Contoreggi C, Matochik J, Kurian V, Cadet J, Kimes A, Funderburk F, London E (2004) Prefrontal cortical dysfunction in abstinent cocaine abusers. J Neuropsychiatry Clin Neurosci 16:456-464. CrossRef Medline

Bossaerts P (2010) Risk and risk prediction error signals in anterior insula. Brain Struct Funct 214:645-653. CrossRef Medline

Büchel C, Holmes AP, Rees G, Friston KJ (1998) Characterizing stimulusresponse functions using nonlinear regressors in parametric fMRI experiments. Neuroimage 8:140-148. CrossRef Medline

Bucholz KK, Cadoret R, Cloninger CR, Dinwiddie SH, Hesselbrock VM, Nurnberger JI Jr, Reich T, Schmidt I, Schuckit MA (1994) A new, semistructured psychiatric interview for use in genetic linkage studies: a report on the reliability of the SSAGA. J Stud Alcohol 55:149-158. Medline

Cauda F, D’Agata F, Sacco K, Duca S, Geminiani G, Vercelli A (2011) Functional connectivity of the insula in the resting brain. Neuroimage 55:8-23. CrossRef Medline

Chambers CD, Bellgrove MA, Stokes MG, Henderson TR, Garavan H, Robertson IH, Morris AP, Mattingley JB (2006) Executive "brake failure" following deactivation of human frontal lobe. J Cogn Neurosci 18:444455. CrossRef Medline

Clark VP, Beatty GK, Anderson RE, Kodituwakku P, Phillips JP, Lane TDR, Kiehl KA, Calhoun VD (2012) Reduced fMRI activity predicts relapse in patients recovering from stimulant dependence. Hum Brain Mapp.

Cloninger CR (1986) A unified biosocial theory of personality and its role in the development of anxiety states. Psychiatr Dev 4:167-226. Medline

Cloninger CR, Przybeck TR, Svrakic DM, Wetzel RD (1994) The Temperament and Character Inventory (TCI): a guide to its development and use. St. Louis: Center for Psychobiology of Personality, Washington University.

Colzato LS, van den Wildenberg WPM, Hommel B (2007) Impaired inhibitory control in recreational cocaine users. PLoS One 2:e1143. CrossRef Medline

Cox RW (1996) AFNI: software for analysis and visualization of functional magnetic resonance neuroimages. Comput Biomed Res 29:162-173. CrossRef Medline

Di Martino A, Scheres A, Margulies DS, Kelly AM, Uddin LQ, Shehzad Z, Biswal B, Walters JR, Castellanos FX, Milham MP (2008) Functional connectivity of human striatum: a resting state FMRI study. Cereb Cortex 18:2735-2747. CrossRef Medline

Elkashef A, Vocci F (2003) Biological markers of cocaine addiction: implications for medications development. Addict Biol 8:123-139. CrossRef Medline

Emeric EE, Brown JW, Boucher L, Carpenter RH, Hanes DP, Harris R, Logan GD, Mashru RN, Paré M, Pouget P, Stuphorn V, Taylor TL, Schall JD. (2007) Influence of history on saccade countermanding performance in humans and macaque monkeys. Vis Res 47:35-49. CrossRef Medline

Euston DR, Gruber AJ, McNaughton BL (2012) The role of medial prefrontal cortex in memory and decision making. Neuron 76:1057-1070. CrossRef Medline

Fillmore MT, Rush CR (2002) Impaired inhibitory control of behavior in chronic cocaine users. Drug Alcohol Depend 66:265-273. CrossRef Medline
Fu LP, Bi GH, Zou ZT, Wang Y, Ye EM, Ma L, Ming-Fan, Yang Z (2008) Impaired response inhibition function in abstinent heroin dependents: an fMRI study. Neurosci Lett 438:322-326. CrossRef Medline

Goldstein RZ, Volkow ND (2011) Dysfunction of the prefrontal cortex in addiction: neuroimaging findings and clinical implications. Nat Rev Neurosci 12:652-669. CrossRef Medline

Hanes DP, Schall JD (1995) Countermanding saccades in macaque. Vis Neurosci 12:929-937. Medline

Hayden BY, Heilbronner SR, Pearson JM, Platt ML (2011) Surprise signals in anterior cingulate cortex: neuronal encoding of unsigned reward prediction errors driving adjustment in behavior. J Neurosci 31:4178-4187. CrossRef Medline

Hester R, Garavan H (2004) Executive dysfunction in cocaine addiction: evidence for discordant frontal, cingulate, and cerebellar activity. J Neurosci 24:11017-11022. CrossRef Medline

Hester R, Simões-Franklin C, Garavan H (2007) Post-error behavior in active cocaine users: poor awareness of errors in the presence of intact performance adjustments. Neuropsychopharmacology 32:1974-1984. CrossRef Medline

Ide JS, Shenoy P, Yu AJ, Li CS (2013) Bayesian prediction and evaluation in the anterior cingulate cortex. J Neurosci 33:2039-2047. CrossRef Medline

Kaufman JN, Ross TJ, Stein EA, Garavan H (2003) Cingulate hypoactivity in cocaine users during a GO-NOGO task as revealed by event-related functional magnetic resonance imaging. J Neurosci 23:7839-7843. Medline

Kemmotsu N, Villalobos ME, Gaffrey MS, Courchesne E, Müller RA (2005) Activity and functional connectivity of inferior frontal cortex associated with response conflict. Brain Res Cogn Brain Res 24:335-342. CrossRef Medline

Kennerley SW, Behrens TE, Wallis JD (2011) Double dissociation of value computations in orbitofrontal and anterior cingulate neurons. Nat Neurosci 14:1581-1589. CrossRef Medline

Kim YT, Lee SW, Kwon DH, Seo JH, Ahn BC, Lee J (2009) Dose-dependent frontal hypometabolism on FDG-PET in methamphetamine abusers. J Psychiatr Res 43:1166-1170. CrossRef Medline

Lawrence AJ, Luty J, Bogdan NA, Sahakian BJ, Clark L (2009) Impulsivity and response inhibition in alcohol dependence and problem gambling. Psychopharmacology 207:163-172. CrossRef Medline

Li CS, Huang C, Constable RT, Sinha R (2006) Imaging response inhibition in a stop-signal task: neural correlates independent of signal monitoring and post-response processing. J Neurosci 26:186-192. CrossRef Medline

Li CS, Huang C, Yan P, Bhagwagar Z, Milivojevic V, Sinha R (2008) Neural correlates of impulse control during stop signal inhibition in cocainedependent men. Neuropsychopharmacology 33:1798-1806. CrossRef Medline

Logan GD, Cowan WB (1984) On the ability to inhibit thought and action: A theory of an act of control. Psychol Rev 91:295. CrossRef

London ED, Simon SL, Berman SM, Mandelkern MA, Lichtman AM, Bramen J, Shinn AK, Miotto K, Learn J, Dong Y, Matochik JA, Kurian V, Newton T, Woods R, Rawson R, Ling W (2004) Mood disturbances and regional cerebral metabolic abnormalities in recently abstinent methamphetamine abusers. Arch Gen Psychiatry 61:73-84. CrossRef Medline

Margulies DS, Kelly AM, Uddin LQ, Biswal BB, Castellanos FX, Milham MP (2007) Mapping the functional connectivity of anterior cingulate cortex. Neuroimage 37:579-588. CrossRef Medline

Matthews SC, Simmons AN, Arce E, Paulus MP (2005) Dissociation of inhibition from error processing using a parametric inhibitory task during functional magnetic resonance imaging. Neuroreport 16:755-760. CrossRef Medline

Monterosso JR, Aron AR, Cordova X, Xu J, London ED (2005) Deficits in response inhibition associated with chronic methamphetamine abuse. Drug Alcohol Depend 79:273-277. CrossRef Medline

Murray GK, Corlett PR, Clark L, Pessiglione M, Blackwell AD, Honey G, Jones PB, Bullmore ET, Robbins TW, Fletcher PC (2008) Substantia nigra/ventral tegmental reward prediction error disruption in psychosis. Mol Psychiatry 13:239, 267-276. CrossRef Medline

O'Doherty JP (2004) Reward representations and reward-related learning in the human brain: insights from neuroimaging. Curr Opin Neurobiol 14:769-776. CrossRef Medline

O'Reilly JX, Schüffelgen U, Cuell SF, Behrens TE, Mars RB, Rushworth MF (2013) Dissociable effects of surprise and model update in parietal and anterior cingulate cortex. Proc Natl Acad Sci U S A 110:E3660-E3669. CrossRef Medline 
Paré M, Hanes DP (2003) Controlled movement processing: superior colliculus activity associated with countermanded saccades. J Neurosci 23: 6480-6489. Medline

Paulus MP, Hozack N, Frank L, Brown GG, Schuckit MA (2003) Decision making by methamphetamine-dependent subjects is associated with error-rate-independent decrease in prefrontal and parietal activation. Biol Psychiatry 53:65-74. CrossRef Medline

Paulus MP, Tapert SF, Schuckit MA (2005) Neural activation patterns of methamphetamine-dependent subjects during decision making predict relapse. Arch Gen Psychiatry 62:761-768. CrossRef Medline

Paulus MP, Lovero KL, Wittmann M, Leland DS (2008) Reduced behavioral and neural activation in stimulant users to different error rates during decision making. Biol Psychiatry 63:1054-1060. CrossRef Medline

Pinheiro J, Bates D, DebRoy S, Sarkar D (2011) The R Development Core Team 2011 nlme: linear and nonlinear mixed effects models. R package version 3.1-102. Vienna, Austria: R Foundation for Statistical Computing. Available at: http://cran.r-project.org/web/packages/nlme/index.html.

Preuschoff K, Quartz SR, Bossaerts P (2008) Human insula activation reflects risk prediction errors as well as risk. J Neurosci 28:2745-2752. CrossRef Medline

Reske M, Delis DC, Paulus MP (2011) Evidence for subtle verbal fluency deficits in occasional stimulant users: quick to play loose with verbal rules. J Psychiatr Res 45:361-368. CrossRef Medline

Ridderinkhof KR, van den Wildenberg WP, Segalowitz SJ, Carter CS (2004) Neurocognitive mechanisms of cognitive control: the role of prefrontal cortex in action selection, response inhibition, performance monitoring, and reward-based learning. Brain Cogn 56:129-140. CrossRef Medline

Rushworth MF, Behrens TE (2008) Choice, uncertainty and value in prefrontal and cingulate cortex. Nat Neurosci 11:389-397. CrossRef Medline

Salo R, Nordahl TE, Possin K, Leamon M, Gibson DR, Galloway GP, Flynn NM, Henik A, Pfefferbaum A, Sullivan EV (2002) Preliminary evidence of reduced cognitive inhibition in methamphetamine-dependent individuals. Psychiatry Res 111:65-74. CrossRef Medline

Scangos KW, Stuphorn V (2010) Medial frontal cortex motivates but does not control movement initiation in the countermanding task. J Neurosci 30:1968-1982. CrossRef Medline

Schall JD, Stuphorn V, Brown JW (2002) Monitoring and control of action by the frontal lobes. Neuron 36:309-322. CrossRef Medline

Seo H, Lee D (2007) Temporal filtering of reward signals in the dorsal anterior cingulate cortex during a mixed-strategy game. J Neurosci 27:83668377. CrossRef Medline

Shenoy P, Yu AJ (2011) Rational decision-making in inhibitory control. Front Hum Neurosci 5.

Shenoy P, Rao RPN, Yu A (2011) A rational decision making framework for inhibitory control. Adv Neural Inf Process Syst 24.

Simon SL, Domier CP, Sim T, Richardson K, Rawson RA, Ling W (2002) Cognitive performance of current methamphetamine and cocaine abusers. J Addict Dis 21:61-74. CrossRef Medline

Singer T, Critchley HD, Preuschoff K (2009) A common role of insula in feelings, empathy and uncertainty. Trends Cogn Sci 13:334-340. CrossRef Medline
Somerville LH, Heatherton TF, Kelley WM (2006) Anterior cingulate cortex responds differentially to expectancy violation and social rejection. Nat Neurosci 9:1007-1008. CrossRef Medline

Stewart JL, Flagan TM, May AC, Reske M, Simmons AN, Paulus MP (2013) Young adults at risk for stimulant dependence show reward dysfunction during reinforcement-based decision making. Biol Psychiatry 73:235241. CrossRef Medline

Stuphorn V, Schall JD (2006) Executive control of countermanding saccades by the supplementary eye field. Nat Neurosci 9:925-931. CrossRef Medline

Stuphorn V, Brown JW, Schall JD (2010) Role of supplementary eye field in saccade initiation: executive, not direct, control. J Neurophysiol 103:801816. CrossRef Medline

Sugiura M, Kawashima R, Nakagawa M, Okada K, Sato T, Goto R, Sato K, Ono S, Schormann T, Zilles K, Fukuda H (2000) Correlation between human personality and neural activity in cerebral cortex. Neuroimage 11:541-546. CrossRef Medline

Tabibnia G, Monterosso JR, Baicy K, Aron AR, Poldrack RA, Chakrapani S, Lee B, London ED (2011) Different forms of self-control share a neurocognitive substrate. J Neurosci 31:4805-4810. CrossRef Medline

Tapert SF, Granholm E, Leedy NG, Brown SA (2002) Substance use and withdrawal: neuropsychological functioning over 8 years in youth. J Int Neuropsychol Soc 8:873-883. Medline

Verbruggen F, Logan GD (2009) Models of response inhibition in the stopsignal and stop-change paradigms. Neurosci Biobehav Rev 33:647-661. CrossRef Medline

Verdejo-García AJ, López-Torrecillas F, Aguilar de Arcos F, Perez-Garcia M (2005) Differential effects of MDMA, cocaine, and cannabis use severity on distinctive components of the executive functions in polysubstance users: a multiple regression analysis. Addict Behav 30:89-101. CrossRef Medline

Volkow ND, Fowler JS, Wang GJ (1999) Imaging studies on the role of dopamine in cocaine reinforcement and addiction in humans. J Psychopharmacol 13:337-345. CrossRef Medline

Wechsler D (2001) Wechsler Test of Adult Reading: WTAR: Psychological Corporation.

White NM (1997) Mnemonic functions of the basal ganglia. Curr Opin Neurobiol 7:164-169. CrossRef Medline

Yoshida W, Ishii S (2006) Resolution of uncertainty in prefrontal cortex. Neuron 50:781-789. CrossRef Medline

Yu A, Cohen J (2009) Sequential effects: Superstition or rational behavior. Advances in Neural Information Processing Systems 21:1873-1880.

Yu AJ, Dayan P (2005) Uncertainty, neuromodulation, and attention. Neuron 46:681-692. CrossRef Medline

Zago L, Petit L, Turbelin MR, Andersson F, Vigneau M, Tzourio-Mazoyer N (2008) How verbal and spatial manipulation networks contribute to calculation: an fMRI study. Neuropsychologia 46:2403-2414. CrossRef Medline

Zuckerman M, Link K (1968) Construct validity for the sensation-seeking scale. J Consult Clin Psychol 32:420-426. CrossRef Medline 